\title{
Higher-Stage Noether Identities and Second Noether Theorems
}

\author{
G. Sardanashvily \\ Department of Theoretical Physics, Moscow State University, Moscow 119999, Russia \\ Correspondence should be addressed to G. Sardanashvily; sardanashvi@phys.msu.ru
}

Received 16 February 2015; Accepted 1 June 2015

Academic Editor: Kamil Brádler

Copyright (C) 2015 G. Sardanashvily. This is an open access article distributed under the Creative Commons Attribution License, which permits unrestricted use, distribution, and reproduction in any medium, provided the original work is properly cited.

\begin{abstract}
The direct and inverse second Noether theorems are formulated in a general case of reducible degenerate Grassmann-graded Lagrangian theory of even and odd variables on graded bundles. Such Lagrangian theory is characterized by a hierarchy of nontrivial higher-stage Noether identities which is described in the homology terms. If a certain homology regularity condition holds, one can associate with a reducible degenerate Lagrangian the exact Koszul-Tate chain complex possessing the boundary operator whose nilpotentness is equivalent to all complete nontrivial Noether and higher-stage Noether identities. The second Noether theorems associate with the above-mentioned Koszul-Tate complex a certain cochain sequence whose ascent operator consists of the gauge and higher-order gauge symmetries of a Lagrangian system. If gauge symmetries are algebraically closed, this operator is extended to the nilpotent BRST operator which brings the above-mentioned cochain sequence into the BRST complex and provides a BRST extension of an original Lagrangian.
\end{abstract}

\section{Introduction}

The second Noether theorems are well known to provide the correspondence between Noether identities (henceforth NI) and gauge symmetries of a Lagrangian system [1]. We aim to formulate these theorems in a general case of reducible degenerate Lagrangian systems characterized by a hierarchy of nontrivial higher-stage NI [2,3]. To describe this hierarchy, one needs to involve Grassmann-graded objects. In a general setting, we therefore consider Grassmann-graded Lagrangian systems of even and odd variables on a smooth manifold $X$ (Section 5).

Lagrangian theory of even (commutative) variables on an $n$-dimensional smooth manifold $X$ conventionally is formulated in terms of smooth fibre bundles over $X$ and jet manifolds of their sections [3-5] in the framework of general technique of nonlinear differential operators and equations $[3,6,7]$. At the same time, different geometric models of odd variables either on graded manifolds or supermanifolds are discussed [8-12]. Both graded manifolds and supermanifolds are phrased in terms of sheaves of graded commutative algebras [12, 13]. However, graded manifolds are characterized by sheaves on smooth manifolds, while supermanifolds are constructed by gluing of sheaves on supervector spaces. Since nontrivial higher-stage NI of a
Lagrangian system on a smooth manifold $X$ form graded $C^{\infty}(X)$-modules, we follow the well known Serre-Swan theorem extended to graded manifolds (Theorem 5) [12]. It states that if a graded commutative $C^{\infty}(X)$-ring is generated by a projective $C^{\infty}(X)$-module of finite rank, it is isomorphic to a ring of graded functions on a graded manifold whose body is $X$. Accordingly, we describe odd variables in terms of graded manifolds $[3,12]$.

Let us recall that a graded manifold is a locally ringed space, characterized by a smooth body manifold $Z$ and some structure sheaf $\mathfrak{A}$ of Grassmann algebras on $Z[12,13]$. Its sections form a graded commutative $C^{\infty}(Z)$-ring of graded functions on a graded manifold $(Z, \mathfrak{A})$. The differential calculus on a graded manifold is defined as the ChevalleyEilenberg differential calculus over this ring (Section 2). By virtue of Batchelor's theorem (Theorem 4), there exists a vector bundle $E \rightarrow Z$ with a typical fibre $V$ such that the structure sheaf $\mathfrak{A}$ of $(Z, \mathfrak{A})$ is isomorphic to a sheaf $\mathfrak{\mathfrak { A }}_{E}$ of germs of sections of the exterior bundle $\wedge E^{*}$ of the dual $E^{*}$ of $E$ whose typical fibre is the Grassmann algebra $\wedge V^{*}[13]$. This Batchelor's isomorphism is not canonical. In applications, it however is fixed from the beginning. Therefore, we restrict our consideration to graded manifolds $\left(Z, \mathfrak{\mathfrak { A }}_{E}\right)$, called the simple graded manifolds (Section 3). 
Lagrangian theory on fibre bundles $Y \rightarrow X$ can be adequately formulated in algebraic terms of a variational bicomplex of exterior forms on the infinite order jet manifold $J^{\infty} Y$ of sections of $Y \rightarrow X$, without appealing to the calculus of variations $[3-5,14]$. This technique is extended to Lagrangian theory on graded manifolds and bundles $[2,12,15,16]$. It is phrased in terms of the Grassmanngraded variational bicomplex of graded exterior forms on a graded infinite order jet manifold $\left(J^{\infty} Y, \mathscr{A}_{J^{\infty} F}\right)$ (Section 5). Lagrangians and the Euler-Lagrange operator are defined as elements (63) and the coboundary operator (64) of this bicomplex, respectively.

A problem is that any Euler-Lagrange operator satisfies NI, which therefore must be separated into the trivial and nontrivial ones. These NI can obey first-stage NI, which in turn are subject to the second-stage ones, and so on. Thus, there is a hierarchy of NI and higher-stage NI which must be separated into the trivial and nontrivial ones (Section 7). If certain homology regularity conditions hold (Condition 1), one can associate with a Lagrangian system the exact KoszulTate (henceforth KT) complex (123) possessing the boundary $\mathrm{KT}$ operator whose nilpotentness is equivalent to all complete nontrivial NI (99) and higher-stage NI (124) [2, 12].

The inverse second Noether theorem formulated in homology terms (Theorem 33) associates with this KT complex (123) the cochain sequence (138) with the ascent operator (139), called the gauge operator, whose components are nontrivial gauge and higher-stage gauge symmetries of Lagrangian theory $[2,12]$. Conversely, given these symmetries, the direct second Noether theorem (Theorem 34) states that the corresponding NI and higher-stage NI hold.

The gauge operator unlike the KT one is not nilpotent, and gauge symmetries need not form an algebra [17-19]. Gauge symmetries are said to be algebraically closed if the gauge operator admits the nilpotent BRST extension (155). If this extension exists, the above-mentioned cochain sequence (138) is brought into the BRST complex (156). The KT and BRST complexes provide the BRST extension (177) of an original Lagrangian theory by antifields and ghosts $[12,18]$.

The most physically relevant Yang-Mills gauge theory on principal bundles and gauge gravitation theory on natural bundles are irreducible degenerate Lagrangian systems which possess nontrivial Noether identities, but trivial first-stage ones $[2,20]$. In Section 10, we analyze topological BF theory which exemplifies a finitely reducible degenerate Lagrangian model.

Remark 1. Smooth manifolds throughout are assumed to be Hausdorff, second-countable, and, consequently, paracompact. Given a smooth manifold $X$, its tangent and cotangent bundles $T X$ and $T^{*} X$ are endowed with bundle coordinates $\left(x^{\lambda}, \dot{x}^{\lambda}\right)$ and $\left(x^{\lambda}, \dot{x}_{\lambda}\right)$ with respect to holonomic frames $\left\{\partial_{\lambda}\right\}$ and $\left\{d x^{\lambda}\right\}$, respectively. Given a coordinate chart $\left(U ; x^{\lambda}\right)$ of $X$, a multi-index $\Lambda$ of length $|\Lambda|=k$ denotes a collection of indices $\left(\lambda_{1} \cdots \lambda_{k}\right)$ modulo permutations. By $\lambda+\Lambda$ is meant a multi-index $\left(\lambda \lambda_{1} \cdots \lambda_{k}\right)$. We use the compact notation $\partial_{\Lambda}=$ $\partial_{\lambda_{k}} \circ \cdots \circ \partial_{\lambda_{1}}$

\section{Grassmann-Graded Differential Calculus}

Throughout this work, by the Grassmann gradation is meant the $\mathbb{Z}_{2}$-one, and a Grassmann-graded structure is called graded if there is no danger of confusion. The symbol [.] stands for the Grassmann parity. Let us recall the relevant basics of the graded algebraic calculus $[12,13]$.

Let $\mathscr{K}$ be a commutative ring. A $\mathscr{K}$-module $Q$ is called graded if it is endowed with a grading automorphism $\gamma, \gamma^{2}=$ Id. A graded module falls into a direct sum of modules $Q=$ $Q_{0} \oplus Q_{1}$ such that $\gamma(q)=(-1)^{[q]} q, q \in Q_{[q]}$. One calls $Q_{0}$ and $Q_{1}$ the even and odd parts of $Q$, respectively. In particular, by a real graded vector space $B=B_{0} \oplus B_{1}$ is meant a graded $\mathbb{R}$-module.

A $\mathscr{K}$-algebra $\mathscr{A}$ is called graded if it is a graded $\mathscr{K}$-module such that $\left[a a^{\prime}\right]=[a]+\left[a^{\prime}\right]$, where $a$ and $a^{\prime}$ are gradedhomogeneous elements of $\mathscr{A}$. Its even part $\mathscr{A}_{0}$ is a subalgebra of $\mathscr{A}$, and the odd one $\mathscr{A}_{1}$ is an $\mathscr{A}_{0}$-module. If $\mathscr{A}$ is a graded ring with the unit 1 , then $[\mathbf{1}]=0$. A graded algebra $\mathscr{A}$ is called graded commutative if $a a^{\prime}=(-1)^{[a]\left[a^{\prime}\right]} a^{\prime} a$.

Hereafter, all algebras and vector spaces are assumed to be real.

Remark 2. Let $V$ be a vector space and $\Lambda=\wedge V$ its exterior algebra. It is a graded commutative ring, called the Grassmann algebra, with respect to the Grassmann gradation

$$
\begin{aligned}
\Lambda & =\Lambda_{0} \oplus \Lambda_{1}, \\
\Lambda_{0} & =\mathbb{R} \bigoplus_{1 \leq k}^{2 k} \bigwedge^{2 k} V, \\
\Lambda_{1} & =\bigoplus_{1 \leq k} \bigwedge^{2 k-1} V .
\end{aligned}
$$

Hereafter, Grassmann algebras of finite rank when $V=\mathbb{R}^{N}$ only are considered.

Given a graded algebra $\mathscr{A}$, a left graded $\mathscr{A}$-module $Q$ is defined as a left $\mathscr{A}$-module where $[a q]=[a]+[q]$. Similarly, right graded $\mathscr{A}$-modules are treated. If $\mathscr{A}$ is graded commutative, a graded $\mathscr{A}$-module $Q$ is provided with a graded $\mathscr{A}$-bimodule structure by letting $q a=(-1)^{[a][q]} a q$.

Remark 3. A graded algebra $\mathfrak{g}$ is called a Lie superalgebra if its product $[\cdot, \cdot]$, called the Lie superbracket, obeys the relations

$$
\begin{aligned}
& {\left[\varepsilon, \varepsilon^{\prime}\right]=-(-1)^{[\varepsilon]\left[\varepsilon^{\prime}\right]}\left[\varepsilon^{\prime}, \varepsilon\right],} \\
& (-1)^{[\varepsilon]\left[\varepsilon^{\prime \prime}\right]}\left[\varepsilon,\left[\varepsilon^{\prime}, \varepsilon^{\prime \prime}\right]\right]+(-1)^{\left[\varepsilon^{\prime}\right][\varepsilon]}\left[\varepsilon^{\prime},\left[\varepsilon^{\prime \prime}, \varepsilon\right]\right] \\
& +(-1)^{\left[\varepsilon^{\prime \prime}\right]\left[\varepsilon^{\prime}\right]}\left[\varepsilon^{\prime \prime},\left[\varepsilon, \varepsilon^{\prime}\right]\right]=0 .
\end{aligned}
$$

A graded vector space $P$ is a $\mathfrak{g}$-module if it is provided with an $\mathbb{R}$-bilinear map

$$
\begin{aligned}
\mathfrak{g} \times P & \ni(\varepsilon, p) \longrightarrow \varepsilon p \in P, \\
{[\varepsilon p] } & =[\varepsilon]+[p], \\
{\left[\varepsilon, \varepsilon^{\prime}\right] p } & =\left(\varepsilon \circ \varepsilon^{\prime}-(-1)^{[\varepsilon]\left[\varepsilon^{\prime}\right]} \varepsilon^{\prime} \circ \varepsilon\right) p .
\end{aligned}
$$


Given a graded commutative ring $\mathscr{A}$, the following are standard constructions of new graded modules from the old ones.

(i) The direct sum of graded modules and a graded factor module are defined just as those of modules over a commutative ring.

(ii) The tensor product $P \otimes Q$ of graded $\mathscr{A}$-modules $P$ and $Q$ is their tensor product as $\mathscr{A}$-modules such that

$$
\begin{aligned}
& {[p \otimes q]=[p]+[q],} \\
& a p \otimes q=(-1)^{[p][a]} p a \otimes q=(-1)^{[p][a]} p \otimes a q .
\end{aligned}
$$

In particular, the tensor algebra $\otimes P$ of a graded $\mathscr{A}$-module $P$ is defined just as that of a module over a commutative ring. Its quotient $\wedge P$ with respect to the ideal generated by elements

$$
p \otimes p^{\prime}+(-1)^{[p]\left[p^{\prime}\right]} p^{\prime} \otimes p, \quad p, p^{\prime} \in P,
$$

is a bigraded exterior algebra of a graded module $P$ provided with a graded exterior product

$$
p \wedge p^{\prime}=-(-1)^{[p]\left[p^{\prime}\right]} p^{\prime} \wedge p .
$$

(iii) A morphism $\Phi: P \rightarrow Q$ of graded $\mathscr{A}$-modules seen as additive groups is said to be an even (resp., odd) graded morphism if $\Phi$ preserves (resp., changes) the Grassmann parity of all graded-homogeneous elements of $P$ and if the relations

$$
\Phi(a p)=(-1)^{[\Phi][a]} a \Phi(p), \quad p \in P, a \in \mathscr{A},
$$

hold. A morphism $\Phi: P \rightarrow Q$ of graded $\mathscr{A}$-modules as additive groups is called a graded $\mathscr{A}$-module morphism if it is represented by a sum of even and odd graded morphisms. A set $\operatorname{Hom}_{\mathscr{A}}(P, Q)$ of graded morphisms of a graded $\mathscr{A}$-module $P$ to a graded $\mathscr{A}$-module $Q$ is naturally a graded $\mathscr{A}$-module. A graded $\mathscr{A}$-module $P^{*}=\operatorname{Hom}_{\mathscr{A}}(P, \mathscr{A})$ is called the dual of P.

Linear differential operators and the differential calculus over a graded commutative ring are defined similarly to those in commutative geometry [3].

Let $\mathscr{A}$ be a graded commutative ring and $P, Q$ graded $\mathscr{A}$-modules. A vector space $\operatorname{Hom}(P, Q)$ of graded real space homomorphisms $\Phi: P \rightarrow Q$ admits two graded $\mathscr{A}$-module structures

$$
\begin{aligned}
(a \Phi)(p) & =a \Phi(p), \\
(\Phi \star a)(p) & =\Phi(a p),
\end{aligned}
$$

$$
a \in \mathscr{A}, p \in P \text {. }
$$

Let us put

$$
\delta_{a} \Phi=a \Phi-(-1)^{[a][\Phi]} \Phi \star a, \quad a \in \mathscr{A} .
$$

An element $\Delta \in \operatorname{Hom}(P, Q)$ is said to be a $Q$-valued graded differential operator of order $s$ on $P$ if $\delta_{a_{0}} \circ \cdots \circ \delta_{a_{s}} \Delta=0$ for any tuple of $s+1$ elements $a_{0}, \ldots, a_{s}$ of $\mathscr{A}$.

In particular, zero order graded differential operators are $\mathscr{A}$-module morphisms $P \rightarrow Q$. For instance, let $P=\mathscr{A}$.
Any zero order $Q$-valued graded differential operator $\Delta$ on $\mathscr{A}$ is given by its value $\Delta(\mathbf{1})$. A first order $Q$-valued graded differential operator $\Delta$ on $\mathscr{A}$ obeys a condition

$$
\begin{aligned}
\Delta(a b)= & \Delta(a) b+(-1)^{[a][\Delta]} a \Delta(b) \\
& -(-1)^{([b]+[a])[\Delta]} a b \Delta(\mathbf{1}), \quad a, b \in \mathscr{A} .
\end{aligned}
$$

It is called the $Q$-valued graded derivation of $\mathscr{A}$ if $\Delta(\mathbf{1})=0$; that is, the graded Leibniz rule

$$
\Delta(\mathrm{a} b)=\Delta(a) b+(-1)^{[a][\Delta]} a \Delta(b), \quad a, b \in \mathscr{A},
$$

holds. If $\partial$ is a graded derivation of $\mathscr{A}$, then $a \partial$ is so for any $a \in \mathscr{A}$. Hence, graded derivations of $\mathscr{A}$ constitute a graded $\mathscr{A}$-module $\mathfrak{D}(\mathscr{A}, Q)$, called the graded derivation module. If $\mathrm{Q}=\mathscr{A}$, a graded derivation module $\boldsymbol{D} \mathscr{A}$ also is a real Lie superalgebra with respect to a superbracket

$$
\left[u, u^{\prime}\right]=u \circ u^{\prime}-(-1)^{[u]\left[u^{\prime}\right]} u^{\prime} \circ u, \quad u, u^{\prime} \in \mathscr{A} .
$$

Since $\mathfrak{d} \mathscr{A}$ is a Lie superalgebra, let us consider the Chevalley-Eilenberg complex $C^{*}[\mathfrak{d} \mathscr{A} ; \mathscr{A}]$, where a graded commutative ring $\mathscr{A}$ is regarded as a $\mathfrak{D} \mathscr{A}$-module $[3,21]$. It is a complex

$$
\begin{aligned}
& 0 \longrightarrow \mathbb{R} \longrightarrow \mathscr{A} \stackrel{d}{\longrightarrow} C^{1}[\mathfrak{d} \mathscr{A} ; \mathscr{A}] \stackrel{d}{\longrightarrow} \cdots C^{k}[\mathfrak{d} \mathscr{A} ; \mathscr{A}] \\
& \quad \stackrel{d}{\longrightarrow} \cdots
\end{aligned}
$$

where $C^{k}[\mathfrak{d} \mathscr{A} ; \mathscr{A}]=\operatorname{Hom}\left(\wedge^{k} \mathfrak{d} \mathscr{A}, \mathscr{A}\right)$ are $\mathfrak{d} \mathscr{A}$-modules of real linear graded morphisms of graded exterior products $\wedge^{k} \mathfrak{D} \mathscr{A}$ to $\mathscr{A}$. One can show that complex (13) contains a subcomplex $\mathcal{O}^{*}[\mathfrak{D} \mathscr{A}]$ of $\mathscr{A}$-linear graded morphisms [3]. The $\mathbb{N}$-graded module $\mathcal{O}^{*}[\mathfrak{d} \mathscr{A}]$ is provided with the structure of a bigraded $\mathscr{A}$-algebra with respect to the graded exterior product

$$
\begin{aligned}
& \phi \wedge \phi^{\prime}\left(u_{1}, \ldots, u_{r+s}\right) \\
&= \sum_{i_{1}<\cdots<i_{r} ; j_{1}<\cdots<j_{s}} \operatorname{Sgn}_{1 \cdots r+s}^{i_{1} \cdots i_{r} j_{1} \cdots j_{s}} \phi\left(u_{i_{1}}, \ldots, u_{i_{r}}\right) \\
& \cdot \phi^{\prime}\left(u_{j_{1}}, \ldots, u_{j_{s}}\right),
\end{aligned}
$$

where $\phi \in \mathcal{O}^{r}[\mathfrak{d} \mathscr{A}], \phi^{\prime} \in \mathcal{O}^{s}[\mathfrak{d} \mathscr{A}]$, and $u_{1}, \ldots, u_{r+s}$ are gradedhomogeneous elements of $\mathfrak{d} \mathscr{A}$. The Chevalley-Eilenberg coboundary operator $d(13)$ and the exterior product $\wedge$ (14) obey relations

$$
\begin{gathered}
\phi \wedge \phi^{\prime}=(-1)^{|\phi|\left|\phi^{\prime}\right|+[\phi]\left[\phi^{\prime}\right]} \phi^{\prime} \wedge \phi, \\
d\left(\phi \wedge \phi^{\prime}\right)=d \phi \wedge \phi^{\prime}+(-1)^{|\phi|} \phi \wedge d \phi^{\prime},
\end{gathered}
$$

and thus they bring $\mathcal{O}^{*}[\boldsymbol{d} \mathscr{A}]$ into a differential bigraded algebra (henceforth DBGA). It is called the graded differential calculus over a graded commutative ring $\mathscr{A}$. In particular, we have

$$
\mathcal{O}^{1}[\mathfrak{d} \mathscr{A}]=\operatorname{Hom}_{\mathscr{A}}(\mathfrak{d} \mathscr{A}, \mathscr{A})=\mathfrak{d} \mathscr{A}^{*} .
$$


One can extend this duality relation to any element $\phi \epsilon$ $\mathcal{O}^{*}[\mathfrak{d} \mathbb{A}]$ by the rules

$$
\begin{aligned}
u\rfloor(b d a)= & (-1)^{[u][b]} b u(a), \\
u\rfloor\left(\phi \wedge \phi^{\prime}\right)= & (u\rfloor \phi) \wedge \phi^{\prime}+(-1)^{|\phi|+[\phi][u]} \phi \\
& \left.\wedge(u\rfloor \phi^{\prime}\right),
\end{aligned}
$$

$$
a, b \in \mathscr{A} \text {. }
$$

As a consequence, every graded derivation $u \in \mathfrak{d} \mathscr{A}$ of $\mathscr{A}$ yields a derivation

$$
\begin{aligned}
\mathbf{L}_{u} \phi & =u\rfloor d \phi+d(u\rfloor \phi), \\
\mathbf{L}_{u}\left(\phi \wedge \phi^{\prime}\right) & =\mathbf{L}_{u}(\phi) \wedge \phi^{\prime}+(-1)^{[u][\phi]} \phi \wedge \mathbf{L}_{u}\left(\phi^{\prime}\right),
\end{aligned}
$$

called the graded Lie derivative, of the DBGA $\mathscr{O}^{*}[\mathfrak{D} \mathscr{A}]$.

The minimal graded differential calculus $\mathscr{O}^{*} \mathscr{A} \subset \mathscr{O}^{*}[\mathfrak{D} \mathscr{A}]$ over a graded commutative ring $\mathscr{A}$ consists of the monomials $a_{0} d a_{1} \wedge \cdots \wedge d a_{k}, a_{i} \in \mathscr{A}$. The corresponding complex

$$
0 \longrightarrow \mathbb{R} \longrightarrow \mathscr{A} \stackrel{d}{\longrightarrow} \mathcal{O}^{1} \mathscr{A} \stackrel{d}{\longrightarrow} \cdots \mathcal{O}^{k} \mathscr{A} \stackrel{d}{\longrightarrow} \cdots
$$

is called the de Rham complex of a graded commutative ring $\mathscr{A}$.

\section{Graded Manifolds and Bundles}

A graded manifold of dimension $(n, m)$ is defined as a localringed space $(Z, \mathfrak{A})$, where $Z$ is an $n$-dimensional smooth manifold $Z$ and $\mathfrak{A}=\mathfrak{A}_{0} \oplus \mathfrak{A}_{1}$ is a sheaf of Grassmann algebras $\Lambda$ of rank $m$ (Remark 2) such that $[3,13]$ (i) there is the exact sequence of sheaves

$$
0 \longrightarrow \mathscr{R} \longrightarrow \mathfrak{A} \stackrel{\sigma}{\longrightarrow} C_{Z}^{\infty} \longrightarrow 0, \quad \mathscr{R}=\mathfrak{A}_{1}+\left(\mathfrak{\Re}_{1}\right)^{2},
$$

where $\sigma$ is a body epimorphism onto a sheaf $C_{Z}^{\infty}$ of smooth real functions on $Z$; (ii) $\mathscr{R} / \mathscr{R}^{2}$ is a locally free sheaf of $C_{Z}^{\infty}$ modules of finite rank (with respect to pointwise operations), and the sheaf $\mathfrak{A}$ is locally isomorphic to the exterior product $\wedge_{C_{Z}^{\infty}}\left(\mathscr{R} / \mathscr{R}^{2}\right)$.

A sheaf $\mathfrak{A}$ is called the structure sheaf of a graded manifold $(Z, \mathfrak{A})$, and a manifold $Z$ is said to be its body. Sections of a sheaf $\boldsymbol{A}$ are called graded functions on a graded manifold $(Z, \mathfrak{A})$. They constitute a graded commutative $C^{\infty}(Z)$-ring $\mathfrak{A}(Z)$ called the structure ring of $(Z, \mathfrak{A})$.

By virtue of Batchelor's theorem [13,22], graded manifolds possess the following structure.

Theorem 4. Let $(Z, \mathfrak{A})$ be a graded manifold. There exists a vector bundle $E \rightarrow Z$ with an $m$-dimensional typical fibre $V$ so that the structure sheaf of $(Z, \mathfrak{U})$ is isomorphic to a sheaf $\mathfrak{A}_{E}$ of sections of the exterior bundle $\wedge E^{*}$ whose typical fibre is a Grassmann algebra $\wedge V^{*}$.

Combining Theorem 4 and the above-mentioned classical Serre-Swan theorem leads to the following Serre-Swan theorem for graded manifolds [12].
Theorem 5. Let $Z$ be a smooth manifold. A graded commutative $C^{\infty}(Z)$-algebra $\mathscr{A}$ is isomorphic to the structure ring of a graded manifold with a body $Z$ iff it is the exterior algebra of some projective $C^{\infty}(Z)$-module of finite rank.

As was mentioned above Batchelor's isomorphism in Theorem 4 is not canonical, and we agree to call $\left(Z, \mathfrak{A}_{E}\right)$ in Theorem 4 the simple graded manifold modelled over a characteristic vector bundle $E \rightarrow Z$. Accordingly, the structure ring $\mathfrak{A}_{E}(Z)$ of $\left(Z, \mathfrak{A}_{E}\right)$ is a structure module

$$
\mathscr{A}_{E}=\mathfrak{A}_{E}(Z)=\wedge E^{*}(Z)
$$

of sections of the exterior bundle $\wedge E^{*}$.

Remark 6. One can treat a local-ringed space $\left(Z, \mathfrak{A}_{0}=C_{Z}^{\infty}\right)$ as a trivial graded manifold. It is a simple graded manifold whose characteristic bundle is $E=Z \times\{0\}$. Its structure module is a ring $C^{\infty}(Z)$ of smooth real functions on $Z$.

Given a simple graded manifold $\left(Z, \mathfrak{A}_{E}\right)$, every trivialization chart $\left(U ; z^{A}, q^{a}\right)$ of a vector bundle $E \rightarrow Z$ yields a splitting domain $\left(U ; z^{A}, c^{a}\right)$ of $\left(Z, \mathfrak{A}_{E}\right)$ where $\left\{c^{a}\right\}$ is the corresponding local fibre basis for $E^{*} \rightarrow X$. Graded functions on such a chart are $\Lambda$-valued functions

$$
f=\sum_{k=0}^{m} \frac{1}{k !} f_{a_{1} \cdots a_{k}}(z) c^{a_{1}} \cdots c^{a_{k}},
$$

where $f_{a_{1} \cdots a_{k}}(z)$ are smooth functions on $U$. One calls $\left\{z^{A}, c^{a}\right\}$ the local generating basis for a graded manifold $\left(Z, \mathfrak{A}_{E}\right)$. Transition functions $q^{\prime a}=\rho_{b}^{a}\left(z^{A}\right) q^{b}$ of bundle coordinates on $E \rightarrow Z$ induce the corresponding transformation law $c^{\prime a}=\rho_{b}^{a}\left(z^{A}\right) c^{b}$ of the associated local generating basis for a graded manifold $\left(Z, \mathfrak{A}_{E}\right)$.

Let us consider the graded derivation module $\mathfrak{b} \mathfrak{A}(Z)$ of a graded commutative ring $\mathfrak{A}(Z)$. It is a Lie superalgebra relative to superbracket (12). Its elements are called the graded vector fields on a graded manifold $(Z, \mathfrak{A})$. A key point is the following $[3,23]$.

Lemma 7. Graded vector fields $u \in \mathfrak{D} \mathscr{A}_{E}$ on a simple graded manifold $\left(Z, \mathfrak{A}_{E}\right)$ are represented by sections of some vector bundle $\mathscr{V}_{E}$ which is locally isomorphic to $\wedge E^{*} \otimes_{Z}\left(E \otimes_{Z} T Z\right)$.

Graded vector fields on a splitting domain $\left(U ; z^{A}, c^{a}\right)$ of $\left(Z, \mathfrak{A}_{E}\right)$ read

$$
\begin{aligned}
u & =u^{A} \partial_{A}+u^{a} \partial_{a}, \\
\partial_{a} \circ \partial_{b} & =-\partial_{b} \circ \partial_{a}, \\
\partial_{A} \circ \partial_{a} & =\partial_{a} \circ \partial_{A},
\end{aligned}
$$

where $u^{A}, u^{a}$ are local graded functions on $U$ possessing a coordinate transformation law

$$
\begin{aligned}
& u^{\prime A}=u^{A}, \\
& u^{\prime a}=\rho_{j}^{a} u^{j}+u^{A} \partial_{A}\left(\rho_{j}^{a}\right) c^{j} .
\end{aligned}
$$


Graded vector fields act on graded functions $f \in \mathfrak{A}_{E}(U)$ (22) by the rule

$$
\begin{aligned}
u\left(f_{a \cdots b} c^{a} \cdots c^{b}\right)= & u^{A} \partial_{A}\left(f_{a \cdots b}\right) c^{a} \cdots c^{b} \\
& \left.+u^{k} f_{a \cdots b} \partial_{k}\right\rfloor\left(c^{a} \cdots c^{b}\right) .
\end{aligned}
$$

Given a structure ring $\mathscr{A}_{E}$ of graded functions on a simple graded manifold $\left(Z, \mathfrak{A}_{E}\right)$ and the Lie superalgebra $\mathfrak{d} \mathscr{A}_{E}$ of its graded derivations, let us consider the graded differential calculus

$$
\mathcal{S}^{*}[E ; Z]=\mathcal{O}^{*}\left[\mathfrak{d} \mathscr{A}_{E}\right]
$$

over $\mathscr{A}_{E}$ where $\delta^{0}[E ; Z]=\mathscr{A}_{E}$. Since the graded derivation module $\mathfrak{D} \mathscr{A}_{E}$ is isomorphic to the structure module of sections of a vector bundle $\mathscr{V}_{E} \rightarrow Z$ in Lemma 7, elements of $\mathcal{S}^{*}[E ; Z]$ are represented by sections of the exterior bundle $\wedge \overline{\mathscr{V}}_{E}$ of the $\mathscr{A}_{E}$-dual $\overline{\mathscr{V}}_{E} \rightarrow Z$ of $\mathscr{V}_{E}$. With respect to the dual fibre bases $\left\{d z^{A}\right\}$ for $T^{*} Z$ and $\left\{d c^{b}\right\}$ for $E^{*}$, sections of $\overline{\mathscr{V}}_{E}$ take a coordinate form

$$
\begin{aligned}
\phi & =\phi_{A} d z^{A}+\phi_{a} d c^{a}, \\
\phi_{a}^{\prime} & =\rho_{a}^{-1^{b}} \phi_{b}, \\
\phi_{A}^{\prime} & =\phi_{A}+\rho^{-1}{ }_{a}^{b} \partial_{A}\left(\rho_{j}^{a}\right) \phi_{b} c^{j} .
\end{aligned}
$$

The duality isomorphism $\mathcal{S}^{1}[E ; Z]=\mathfrak{d} \mathscr{A}_{E}^{*}(16)$ is given by the graded interior product

$$
u\rfloor \phi=u^{A} \phi_{A}+(-1)^{\left[\phi_{a}\right]} u^{a} \phi_{a} .
$$

Elements of $\mathcal{S}^{*}[E ; Z]$ are called graded exterior forms on a graded manifold $\left(Z, \mathfrak{A}_{E}\right)$. In particular, elements of $\mathcal{S}^{0}[E ; Z]$ are graded functions on $\left(Z, \mathfrak{A}_{E}\right)$.

Seen as an $\mathscr{A}_{E}$-algebra, the DBGA $\mathcal{S}^{*}[E ; Z](26)$ on a splitting domain $\left(U ; z^{A}, c^{a}\right)$ is locally generated by graded one-forms $d z^{A}, d c^{i}$ such that

$$
\begin{gathered}
d z^{A} \wedge d c^{i}=-d c^{i} \wedge d z^{A}, \\
d c^{i} \wedge d c^{j}=d c^{j} \wedge d c^{i} .
\end{gathered}
$$

Accordingly, the graded Chevalley-Eilenberg coboundary operator $d$ (13), called the graded exterior differential, reads

$$
d \phi=d z^{A} \wedge \partial_{A} \phi+d c^{a} \wedge \partial_{a} \phi,
$$

where derivations $\partial_{\lambda}, \partial_{a}$ act on coefficients of graded exterior forms by formula (25), and they are graded commutative with graded forms $d z^{A}$ and $d c^{a}$. Formulas (15)-(18) hold.

Lemma 8. The DBGA $\mathcal{S}^{*}[E ; Z](26)$ is a minimal differential calculus over $\mathscr{A}_{E}$; that is, it is generated by elements $d f, f \in \mathscr{A}_{E}$ $[3,23]$.

The de Rham complex (19) of the minimal graded differential calculus $\mathcal{S}^{*}[E ; Z]$ reads

$$
\begin{aligned}
0 \longrightarrow & \mathbb{R} \longrightarrow \mathscr{A}_{E} \stackrel{d}{\longrightarrow} \delta^{1}[E ; Z] \stackrel{d}{\longrightarrow} \cdots \delta^{k}[E ; Z] \\
& \stackrel{d}{\longrightarrow} \cdots .
\end{aligned}
$$

Given the differential graded algebra $\mathcal{O}^{*}(Z)$ of exterior forms on $Z$, there exists a canonical cochain monomorphism $\mathcal{O}^{*}(Z) \rightarrow \mathcal{S}^{*}[E ; Z]$ of the de Rham complex $\mathcal{O}^{*}(Z)$ to complex (31).

A morphism of graded manifolds $(Z, \mathfrak{A}) \rightarrow\left(Z^{\prime}, \mathfrak{A}^{\prime}\right)$ is defined as that of local-ringed spaces

$$
\begin{gathered}
\phi: Z \longrightarrow Z^{\prime}, \\
\widehat{\Phi}: \mathfrak{A}^{\prime} \longrightarrow \phi_{*} \mathfrak{A},
\end{gathered}
$$

where $\phi$ is a manifold morphism and $\widehat{\Phi}$ is a sheaf morphism of $\mathfrak{A}^{\prime}$ to the direct image $\phi_{*} \mathfrak{A}$ of $\mathfrak{A}$ onto $Z^{\prime}$. Morphism (32) of graded manifolds is called (i) a monomorphism if $\phi$ is an injection and $\widehat{\Phi}$ is an epimorphism and (ii) an epimorphism if $\phi$ is a surjection and $\widehat{\Phi}$ is a monomorphism.

An epimorphism of graded manifolds $(Z, \mathfrak{A}) \rightarrow$ $\left(Z^{\prime}, \mathfrak{A}^{\prime}\right)$, where $Z \rightarrow Z^{\prime}$ is a fibre bundle, is called the graded bundle $[24,25]$. In this case, a sheaf monomorphism $\widehat{\Phi}$ induces a monomorphism of canonical presheaves $\overrightarrow{\mathfrak{A}}^{\prime} \rightarrow$ $\overline{\mathfrak{A}}$, which associates with each open subset $U \subset Z$ the ring of sections of $\mathfrak{A}^{\prime}$ over $\phi(U)$. Accordingly, there is a pull-back monomorphism of the structure rings $\mathfrak{A}^{\prime}\left(Z^{\prime}\right) \rightarrow \mathfrak{A}(Z)$ of graded functions on graded manifolds $\left(Z^{\prime}, \mathfrak{A}^{\prime}\right)$ and $(Z, \mathfrak{A})$.

In particular, let $(Y, \mathfrak{A})$ be a graded manifold whose body $Z=Y$ is a fibre bundle $\pi: Y \rightarrow X$. Let us consider a trivial graded manifold $\left(X, C_{X}^{\infty}\right)$ (Remark 6$)$. Then we have a graded bundle

$$
(Y, \mathfrak{A}) \longrightarrow\left(X, C_{X}^{\infty}\right) .
$$

We agree to call the graded bundle (33) over a trivial graded manifold $\left(X, C_{X}^{\infty}\right)$ the graded bundle over a smooth manifold. Let us denote it by $(X, Y, \mathfrak{A})$. Given a graded bundle $(X, Y, \mathfrak{A})$, the local generating basis for a graded manifold $(Y, \mathfrak{A})$ can be brought into a form $\left(x^{\lambda}, y^{i}, c^{a}\right)$ where $\left(x^{\lambda}, y^{i}\right)$ are bundle coordinates of $Y \rightarrow X$.

Remark 9. Let $Y \rightarrow X$ be a fibre bundle. Then a trivial graded manifold $\left(Y, C_{Y}^{\infty}\right)$ together with a ring monomorphism $C^{\infty}(X) \rightarrow C^{\infty}(Y)$ is the graded bundle $\left(X, Y, C_{Y}^{\infty}\right)$ (33).

Remark 10. A graded manifold $(X, \mathfrak{U})$ itself can be treated as the graded bundle $(X, X, \mathfrak{A})(33)$ associated with the identity smooth bundle $X \rightarrow X$.

Let $E \rightarrow Z$ and $E^{\prime} \rightarrow Z^{\prime}$ be vector bundles and $\Phi: E \rightarrow$ $E^{\prime}$ their bundle morphism over a morphism $\phi: Z \rightarrow Z^{\prime}$. Then every section $s^{*}$ of the dual bundle $E^{\prime *} \rightarrow Z^{\prime}$ defines the pull-back section $\Phi^{*} s^{*}$ of the dual bundle $E^{*} \rightarrow Z$ by the law

$$
\left.\left.v_{z}\right\rfloor \Phi^{*} s^{*}(z)=\Phi\left(v_{z}\right)\right\rfloor s^{*}(\varphi(z)), \quad v_{z} \in E_{z} .
$$

It follows that a bundle morphism $(\Phi, \phi)$ yields a morphism of simple graded manifolds

$$
\left(Z, \mathfrak{A}_{E}\right) \longrightarrow\left(Z^{\prime}, \mathfrak{A}_{E^{\prime}}\right) .
$$


This is a pair $\left(\phi, \widehat{\Phi}=\phi_{*} \circ \Phi^{*}\right)$ of a morphism $\phi$ of body manifolds and the composition $\phi_{*} \circ \Phi^{*}$ of the pull-back $\mathscr{A}_{E^{\prime}} \ni$ $f \rightarrow \Phi^{*} f \in \mathscr{A}_{E}$ of graded functions and the direct image $\phi_{*}$ of a sheaf $\mathfrak{A}_{E}$ onto $Z^{\prime}$. Relative to local bases $\left(z^{A}, c^{a}\right)$ and $\left(z^{\prime A}, c^{\prime a}\right)$ for $\left(Z, \mathfrak{A}_{E}\right)$ and $\left(Z^{\prime}, \mathfrak{A}_{E^{\prime}}\right)$, morphism (35) of simple graded manifolds reads $z^{\prime}=\phi(z), \widehat{\Phi}\left(c^{\prime a}\right)=\Phi_{b}^{a}(z) c^{b}$.

The graded manifold morphism (35) is a monomorphism (resp., epimorphism) if $\Phi$ is a bundle injection (resp., surjection). In particular, the graded manifold morphism (35) is a graded bundle if $\Phi$ is a fibre bundle. Let $\mathscr{A}_{E^{\prime}} \rightarrow \mathscr{A}_{E}$ be the corresponding pull-back monomorphism of the structure rings. By virtue of Lemma 8 it yields a monomorphism of the DBGAs

$$
\mathcal{S}^{*}\left[E^{\prime} ; Z^{\prime}\right] \longrightarrow \mathcal{S}^{*}[E ; Z] .
$$

Let $\left(Y, \mathfrak{A}_{F}\right)$ be a simple graded manifold modelled over a vector bundle $F \rightarrow Y$. This is a graded bundle $\left(X, Y, \mathfrak{A}_{F}\right)$ modelled over a composite bundle

$$
F \longrightarrow Y \longrightarrow X
$$

If $Y \rightarrow X$ is a vector bundle, this is a particular case of graded vector bundles in $[11,24]$ whose base is a trivial graded manifold. The structure ring of graded functions on a simple graded manifold $\left(Y, \boldsymbol{\mathcal { A }}_{F}\right)$ is the graded commutative $C^{\infty}(X)$-ring $\mathscr{A}_{F}=\wedge F^{*}(Y)(21)$. Let the composite bundle (37) be provided with adapted bundle coordinates $\left(x^{\lambda}, y^{i}, q^{a}\right)$ possessing transition functions $x^{\prime \lambda}\left(x^{\mu}\right), y^{\prime i}\left(x^{\mu}, y^{j}\right)$, and $q^{\prime a}=$ $\rho_{b}^{a}\left(x^{\mu}, y^{j}\right) q^{b}$. Then the corresponding local generating basis for a simple graded manifold $\left(Y, \mathfrak{A}_{F}\right)$ is $\left(x^{\lambda}, y^{i}, c^{a}\right)$ together with transition functions $c^{\prime a}=\rho_{b}^{a}\left(x^{\mu}, j^{j}\right) c^{b}$. We call it the local generating basis for a graded bundle $\left(X, Y, \mathfrak{\mathfrak { A }}_{F}\right)$.

\section{Graded Jet Manifolds}

As was mentioned above, Lagrangian theory on a smooth fibre bundle $Y \rightarrow X$ is formulated in terms of the variational bicomplex on jet manifolds $J^{*} Y$ of $Y$. These are fibre bundles over $X$ and, therefore, they can be regarded as trivial graded bundles $\left(X, J^{k} Y, C_{J^{k} Y}^{\infty}\right)$. Then let us describe their partners in the case of graded bundles $\left(Y, \mathfrak{A}_{F}\right) \rightarrow\left(X, C_{X}^{\infty}\right)$ as follows.

Note that, given a graded manifold $(X, \mathfrak{A})$ and its structure ring $\mathscr{A}$, one can define the jet module $J^{1} \mathscr{A}$ of a $C^{\infty}(X)$ ring $\mathscr{A}[3]$. If $\left(X, \mathfrak{A}_{E}\right)$ is a simple graded manifold modelled over a vector bundle $E \rightarrow X$, the jet module $J^{1} \mathscr{A}_{E}$ is a module of global sections of a jet bundle $J^{1}\left(\wedge E^{*}\right)$. A problem is that $J^{1} \mathscr{A}_{E}$ fails to be a structure ring of some graded manifold. For this reason, we have suggested a different construction of jets of graded manifolds, though it is applied only to simple graded manifolds $[12,23]$.

Let $\left(X, \mathscr{A}_{E}\right)$ be a simple graded manifold modelled over a vector bundle $E \rightarrow X$. Let us consider a $k$-order jet manifold $J^{k} E$ of $E$. It is a vector bundle over $X$. Then let $\left(X, \mathscr{A}_{J^{k} E}\right)$ be a simple graded manifold modelled over $J^{k} E \rightarrow X$. We agree to call $\left(X, \mathscr{A}_{J^{k} E}\right)$ the graded $k$-order jet manifold of a simple graded manifold $\left(X, \mathscr{A}_{E}\right)$. Given a splitting domain $\left(U ; x^{\lambda}, c^{a}\right)$ of a graded manifold $\left(Z, \mathscr{A}_{E}\right)$, we have a splitting domain $\left(U ; x^{\lambda}, c^{a}, c_{\lambda}^{a}, c_{\lambda_{1} \lambda_{2}}^{a}, \ldots, c_{\lambda_{1} \cdots \lambda_{k}}^{a}\right)$ of a graded jet manifold $\left(X, \mathscr{A}_{J^{k} E}\right)$.

As was mentioned above, a graded manifold is a particular graded bundle over its body (Remark 10). Then the definition of graded jet manifolds is generalized to graded bundles over smooth manifolds as follows. Let $\left(X, Y, \mathfrak{A}_{F}\right)$ be a graded bundle modelled over the composite bundle (37). It is readily observed that a jet manifold $J^{r} F$ of $F \rightarrow X$ is a vector bundle $J^{r} F \rightarrow J^{r} Y$ coordinated by $\left(x^{\lambda}, y_{\Lambda}^{i}, q_{\Lambda}^{a}\right)$, $0 \leq|\Lambda| \leq r$. Let $\left(J^{r} Y, \mathfrak{A}_{r}=\mathfrak{A}_{J^{r} F}\right)$ be a simple graded manifold modelled over this vector bundle. Its local generating basis is $\left(x^{\lambda}, y_{\Lambda}^{i}, c_{\Lambda}^{a}\right), 0 \leq|\Lambda| \leq r$. We call $\left(J^{r} Y, \mathfrak{A}_{r}\right)$ the graded $r$-order jet manifold of a graded bundle $\left(X, Y, \mathfrak{\mathfrak { A }}_{F}\right)$.

In particular, let $Y \rightarrow X$ be a smooth bundle seen as a trivial graded bundle $\left(X, Y, C_{Y}^{\infty}\right)$ modelled over a composite bundle $Y \times\{0\} \rightarrow Y \rightarrow X$. Then its graded jet manifold is a trivial graded bundle $\left(X, J^{r} Y, C_{J^{r} Y}^{\infty}\right)$, that is, a jet manifold $J^{r} Y$ of $Y$. Thus, the above definition of jets of graded bundles is compatible with the conventional definition of jets of fibre bundles.

Jet manifolds $J^{*} Y$ of a fibre bundle $Y \rightarrow X$ form the inverse sequence

$$
Y \stackrel{\pi}{\longleftarrow} J^{1} Y \longleftarrow \cdots J^{r-1} Y \stackrel{\pi_{r-1}^{r}}{\longleftarrow} J^{r} Y \longleftarrow \cdots,
$$

of affine bundles $\pi_{r-1}^{r}$. One can think of elements of its projective limit $J^{\infty} Y$ as being infinite order jets of sections of $Y \rightarrow X$ identified by their Taylor series at points of $X$. A set $J^{\infty} Y$ is endowed with the projective limit topology which makes $J^{\infty} Y$ a paracompact Fréchet manifold $[3,5]$. It is called the infinite order jet manifold. A bundle coordinate atlas $\left(x^{\lambda}, y^{i}\right)$ of $Y$ provides $J^{\infty} Y$ with the adapted manifold coordinate atlas

$$
\begin{gathered}
\left(x^{\lambda}, y_{\Lambda}^{i}\right), \\
y_{\lambda+\Lambda}^{\prime i}=\frac{\partial x^{\mu}}{\partial x^{\prime \lambda}} d_{\mu} y_{\Lambda}^{\prime i}, \\
d_{\lambda}=\partial_{\lambda}+y_{\lambda}^{i} \partial_{i}+\sum_{0<|\Lambda|} y_{\lambda+\Lambda}^{i} \partial_{i}^{\Lambda} .
\end{gathered}
$$

The inverse sequence (38) of jet manifolds yields the direct sequence of graded differential algebras $\mathcal{O}_{r}^{*}$ of exterior forms on finite order jet manifolds

$$
\begin{aligned}
\mathcal{O}^{*}(X) & \stackrel{\pi^{*}}{\longrightarrow} \mathcal{O}^{*}(Y) \stackrel{\pi_{0}^{1^{*}}}{\longrightarrow} \mathcal{O}_{1}^{*} \longrightarrow \cdots \mathcal{O}_{r-1}^{*} \stackrel{\pi_{r-1}^{r}}{\longrightarrow} \mathcal{O}_{r}^{*} \\
& \longrightarrow \cdots
\end{aligned}
$$

where $\pi_{r-1}^{r}{ }^{*}$ are the pull-back monomorphisms. Its direct limit

$$
\mathcal{O}_{\infty}^{*}=\overleftrightarrow{\lim } \mathcal{O}_{r}^{*}
$$

consists of all exterior forms on finite order jet manifolds modulo the pull-back identification. The $\mathcal{O}_{\infty}^{*}(41)$ is a differential graded algebra which inherits operations of the exterior differential $d$ and exterior product $\wedge$ of exterior algebras $\mathcal{O}_{r}^{*}$. 
Fibre bundles $J^{r+1} Y \rightarrow J^{r} Y(38)$ and the corresponding bundles $J^{r+1} F \rightarrow J^{r} F$ yield graded bundles $\left(J^{r+1} Y, \mathfrak{Q}_{r+1}\right) \rightarrow$ $\left(J^{r} Y, \mathfrak{A}_{r}\right)$ including pull-back monomorphisms of structure rings

$$
\delta_{r}^{0}[F ; Y] \longrightarrow \delta_{r+1}^{0}[F ; Y]
$$

of graded functions on graded manifolds $\left(J^{r} Y, \mathfrak{A}_{r}\right)$ and $\left(J^{r+1} Y, \mathfrak{A}_{r+1}\right)$. As a consequence, we have the inverse sequence of graded manifolds

$$
\begin{aligned}
\left(Y, \mathscr{A}_{F}\right) & \longleftarrow\left(J^{1} Y, \mathfrak{A}_{J^{1} F}\right) \longleftarrow \cdots\left(J^{r-1} Y, \mathfrak{A}_{J^{r-1} F}\right) \\
& \longleftarrow\left(J^{r} Y, \mathfrak{A}_{J^{r} F}\right) \longleftarrow \cdots .
\end{aligned}
$$

One can think of its inverse limit $\left(J^{\infty} Y, \mathscr{A}_{J^{\infty} F}\right)$ as the graded Fréchet manifold whose body is an infinite order jet manifold $J^{\infty} Y$ and whose structure sheaf $\mathscr{A}_{J^{\infty} F}$ is a sheaf of germs of graded functions on graded manifolds $\left(J^{*} Y, \mathfrak{A}_{J^{*} F}\right)[12,23]$.

By virtue of Lemma 8 , the differential calculus $\mathcal{S}_{r}^{*}[F ; Y]$ is minimal. Therefore, the monomorphisms of structure rings (42) yield the pull-back monomorphisms (36) of DBGAs

$$
\pi_{r}^{r+1 *}: \mathcal{S}_{r}^{*}[F ; Y] \longrightarrow \mathcal{S}_{r+1}^{*}[F ; Y] .
$$

As a consequence, we have a direct system of DBGAs

$$
\begin{aligned}
& \mathcal{S}^{*}[F ; Y] \stackrel{\pi^{*}}{\longrightarrow} \mathcal{S}_{1}^{*}[F ; Y] \\
& \stackrel{\pi_{r-1}^{r *}}{\longrightarrow} \mathcal{S}_{r}^{*}[F ; Y] \longrightarrow \cdots \\
& \mathcal{S}_{r-1}^{*}[F ; Y]
\end{aligned}
$$

The DBGA $\mathcal{S}_{\infty}^{*}[F ; Y]$ that we associate with a graded bundle $\left(Y, \mathfrak{A}_{F}\right)$ is defined as the direct limit

$$
\mathcal{S}_{\infty}^{*}[F ; Y]=\overleftrightarrow{\lim } \mathcal{S}_{r}^{*}[F ; Y]
$$

of the direct system (45). It consists of all graded exterior forms $\phi \in \mathcal{S}^{*}\left[F_{r} ; J^{r} Y\right]$ on graded manifolds $\left(J^{r} Y, \mathfrak{A}_{r}\right)$ modulo monomorphisms (44). Its elements obey relations (15).

The cochain monomorphisms $\mathcal{O}_{r}^{*} \rightarrow \mathcal{S}_{r}^{*}[F ; Y]$ provide a monomorphism of the direct system (40) to the direct system (45) and, consequently, a cochain monomorphism $\mathcal{O}_{\infty}^{*} \rightarrow$ $\mathcal{S}_{\infty}^{*}[F ; Y]$.

One can think of elements of $\mathcal{S}_{\infty}^{*}[F ; Y]$ as being graded differential forms on an infinite order jet manifold $J^{\infty} Y$ in the sense that $\mathcal{S}_{\infty}^{*}[F ; Y]$ is a submodule of the structure module of sections of some sheaf on $J^{\infty} Y[12,23]$. In particular, one can restrict $\mathcal{S}_{\infty}^{*}[F ; Y]$ to the coordinate chart (39) of $J^{\infty} Y$ so that $\mathcal{S}_{\infty}^{*}[F ; Y]$ as an $\mathcal{O}_{\infty}^{0}$-algebra is locally generated by the elements

$$
\begin{array}{r}
\left(c_{\Lambda}^{a}, d x^{\lambda}, \theta_{\Lambda}^{a}=d c_{\Lambda}^{a}-c_{\lambda+\Lambda}^{a} d x^{\lambda}, \theta_{\Lambda}^{i}=d y_{\Lambda}^{i}-y_{\lambda+\Lambda}^{i} d x^{\lambda}\right), \\
0 \leq|\Lambda|,
\end{array}
$$

where $c_{\Lambda}^{a}, \theta_{\Lambda}^{a}$ are odd and $d x^{\lambda}, \theta_{\Lambda}^{i}$ are even. We agree to call $\left(y^{i}, c^{a}\right)$ the local generating basis for $\mathcal{S}_{\infty}^{*}[F ; Y]$. Let the collective symbol $s^{A}$ stand for its elements. We further denote $[A]=\left[s^{A}\right]$.
Remark 11. Let $\left(X, Y, \mathfrak{A}_{F}\right)$ and $\left(X, Y^{\prime}, \mathfrak{A}_{F^{\prime}}\right)$ be graded bundles modelled over composite bundles $F \rightarrow Y \rightarrow X$ and $F^{\prime} \rightarrow$ $Y^{\prime} \rightarrow X$, respectively. Let $F \rightarrow F^{\prime}$ be a fibre bundle over a fibre bundle $Y \rightarrow Y^{\prime}$ over $X$. Then we have a graded bundle $\left(X, Y, \mathfrak{A}_{F}\right) \rightarrow\left(X, Y^{\prime}, \mathfrak{A}_{F^{\prime}}\right)$ together with the pullback monomorphism (36) of DBGAs

$$
\mathcal{S}^{*}\left[F^{\prime} ; Y^{\prime}\right] \longrightarrow \mathcal{S}^{*}[F ; Y] .
$$

Let $\left(X, J^{r} Y, \mathfrak{A}_{J^{r} F}\right)$ and $\left(X, J^{r} Y^{\prime}, \mathfrak{A}_{J^{r} F^{\prime}}\right)$ be graded bundles modelled over composite bundles $J^{r} F \rightarrow J^{r} Y \rightarrow X$ and $J^{r} F^{\prime} \rightarrow J^{r} Y^{\prime} \rightarrow X$, respectively. Since $J^{r} F \rightarrow J^{r} F^{\prime}$ is a fibre bundle over a fibre bundle $J^{r} Y \rightarrow J^{r} Y^{\prime}$ over $X$, we also get a graded bundle

$$
\left(X, J^{r} Y, \mathfrak{A}_{J^{r} F}\right) \longrightarrow\left(X, J^{r} Y^{\prime}, \mathfrak{A}_{J^{r} F^{\prime}}\right)
$$

together with the pull-back monomorphism of DBGAs

$$
\mathcal{S}_{r}^{*}\left[F^{\prime} ; Y^{\prime}\right] \longrightarrow \mathcal{S}_{r}^{*}[F ; Y] .
$$

Monomorphisms (48)-(50), $r=1,2, \ldots$, provide a monomorphism of the direct limits

$$
\mathcal{S}_{\infty}^{*}\left[F^{\prime} ; Y^{\prime}\right] \longrightarrow \mathcal{S}_{\infty}^{*}[F ; Y]
$$

of DBGAs $\mathcal{S}_{r}^{*}\left[F^{\prime} ; Y^{\prime}\right]$ and $\mathcal{S}_{r}^{*}[F ; Y], r=0,1,2, \ldots$

Remark 12. Let $\left(X, Y, \mathfrak{A}_{F}\right)$ and $\left(X, Y^{\prime}, \mathfrak{A}_{F^{\prime}}\right)$ be graded bundles modelled over composite bundles $F \rightarrow Y \rightarrow X$ and $F^{\prime} \rightarrow$ $Y^{\prime} \rightarrow X$, respectively. We define their product

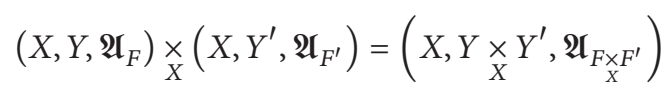

as a graded bundle modelled over a composite bundle

$$
F \underset{X}{\times} F^{\prime}=F \underset{Y \times Y^{\prime}}{\times} F^{\prime} \longrightarrow \underset{X}{Y} \underset{X}{\times} Y^{\prime} \longrightarrow X .
$$

Let us consider the corresponding DBGA

$$
\mathcal{S}_{\infty}^{*}\left[F \underset{X}{\times} F^{\prime} ; Y \underset{X}{\times} Y^{\prime}\right] .
$$

Then, in accordance with Remark 11, there are monomorphisms (51) of BGDAs

$$
\begin{aligned}
& \mathcal{S}_{\infty}^{*}[F ; Y] \longrightarrow \mathcal{S}_{\infty}^{*}\left[F \underset{X}{\times} F ; \underset{X}{\times} Y^{\prime}\right], \\
& \mathcal{S}_{\infty}^{*}\left[F^{\prime} ; Y^{\prime}\right] \longrightarrow \mathcal{S}_{\infty}^{*}\left[F \underset{X}{\times} F ; \underset{X}{\times} Y^{\prime}\right] \text {. }
\end{aligned}
$$

\section{Graded Lagrangian Formalism}

Let $\left(X, Y, \mathfrak{A}_{F}\right)$ be a graded bundle modelled over a composite bundle (37) over an $n$-dimensional smooth manifold $X$, and let $\mathcal{S}_{\infty}^{*}[F ; Y]$ be the associated DBGA (46) of graded exterior forms on graded jet manifolds of $\left(X, Y, \mathfrak{A}_{F}\right)$. As was mentioned above, Grassmann-graded Lagrangian theory of even and odd variables on a graded bundle is formulated 
in terms of the variational bicomplex in which the DBGA $\mathcal{S}_{\infty}^{*}[F ; Y]$ is split in $[2,16,23]$.

A DBGA $\mathcal{S}_{\infty}^{*}[F ; Y]$ is decomposed into $\delta_{\infty}^{0}[F ; Y]$ modules $\delta_{\infty}^{k, r}[F ; Y]$ of $k$-contact and $r$-horizontal graded forms together with the corresponding projections

$$
\begin{aligned}
h_{k}: \mathcal{S}_{\infty}^{*}[F ; Y] & \longrightarrow \mathcal{S}_{\infty}^{k, *}[F ; Y], \\
h^{m}: \mathcal{S}_{\infty}^{*}[F ; Y] & \longrightarrow \mathcal{S}_{\infty}^{*, m}[F ; Y] .
\end{aligned}
$$

Accordingly, the graded exterior differential $d$ on $\mathcal{S}_{\infty}^{*}[F ; Y]$ falls into a sum $d=d_{V}+d_{H}$ of the vertical and total graded differentials

$$
\begin{aligned}
d_{\mathrm{V}} \circ h^{m} & =h^{m} \circ d \circ h^{m}, \\
d_{V}(\phi) & =\theta_{\Lambda}^{A} \wedge \partial_{A}^{\Lambda} \phi, \quad \phi \in \mathcal{S}_{\infty}^{*}[F ; Y], \\
d_{H} \circ h_{k} & =h_{k} \circ d \circ h_{k}, \\
d_{H} \circ h_{0} & =h_{0} \circ d, \\
d_{H}(\phi) & =d x^{\lambda} \wedge d_{\lambda}(\phi), \\
d_{\lambda} & =\partial_{\lambda}+\sum s_{\lambda+\Lambda}^{A} \partial_{A}^{\Lambda},
\end{aligned}
$$

where $d_{\lambda}$ are graded total derivatives. These differentials obey the nilpotent relations

$$
\begin{aligned}
d_{H} \circ d_{H} & =0, \\
d_{V} \circ d_{V} & =0, \\
d_{H} \circ d_{V}+d_{V} \circ d_{H} & =0 .
\end{aligned}
$$

A DBGA $\mathcal{S}_{\infty}^{*}[F ; Y]$ also is provided with the graded projection endomorphism

$$
\begin{aligned}
\varrho & =\sum_{k>0} \frac{1}{k} \bar{\varrho} \circ h_{k} \circ h^{n}: \mathcal{S}_{\infty}^{*>0, n}[F ; Y] \\
& \longrightarrow \mathcal{S}_{\infty}^{*>0, n}[F ; Y], \\
\bar{\varrho}(\phi) & \left.=\sum(-1)^{|\Lambda|} \theta^{A} \wedge\left[d_{\Lambda}\left(\partial_{A}^{\Lambda}\right\rfloor \phi\right)\right], \\
& \quad \phi \in \mathcal{S}_{\infty}^{*>0, n}[F ; Y],
\end{aligned}
$$

such that $\varrho \circ d_{H}=0$, and with the nilpotent graded variational operator

$$
\delta=\varrho \circ d: \delta_{\infty}^{*, n}[F ; Y] \longrightarrow \mathcal{S}_{\infty}^{*+1, n}[F ; Y] .
$$

With these operators a DBGA $\delta_{\infty}^{*}[F ; Y]$ is decomposed into the Grassmann-graded variational bicomplex [12, 23]. We restrict our consideration to the short variational subcomplex

$$
\begin{aligned}
0 \longrightarrow \mathbb{R} \longrightarrow \delta_{\infty}^{0}[F ; Y] \stackrel{d_{H}}{\longrightarrow} \delta_{\infty}^{0,1}[F ; Y] \cdots \\
\stackrel{d_{H}}{\longrightarrow} \delta_{\infty}^{0, n}[F ; Y] \stackrel{\delta}{\longrightarrow} \varrho\left(\mathcal{\delta}_{\infty}^{1, n}[F ; Y]\right)
\end{aligned}
$$

of this bicomplex and its subcomplex of one-contact graded forms

$$
\begin{aligned}
0 \longrightarrow \mathcal{S}_{\infty}^{1,0}[F ; Y] \stackrel{d_{H}}{\longrightarrow} \mathcal{S}_{\infty}^{1,1}[F ; Y] \cdots \stackrel{d_{H}}{\longrightarrow} \mathcal{S}_{\infty}^{1, n}[F ; Y] \\
\stackrel{\varrho}{\longrightarrow} \varrho\left(\mathcal{S}_{\infty}^{1, n}[F ; Y]\right) \longrightarrow 0 .
\end{aligned}
$$

They possess the following cohomology $[12,16]$.

Theorem 13. Cohomology of complex (61) equals the de Rham cohomology of Y. Complex (62) is exact.

Decomposed into a variational bicomplex, the DBGA $\mathcal{S}_{\infty}^{*}[F ; Y]$ describes graded Lagrangian theory on a graded bundle $\left(X, Y, \mathfrak{A}_{F}\right)$. Its graded Lagrangian is defined as an element

$$
L=\mathscr{L} \omega \in \mathcal{S}_{\infty}^{0, n}[F ; Y], \quad \omega=d x^{1} \wedge \cdots \wedge d x^{n},
$$

of the graded variational complex (61). Accordingly, a graded exterior form

$$
\begin{aligned}
\delta L & =\theta^{A} \wedge \mathscr{E}_{A} \omega=\sum(-1)^{|\Lambda|} \theta^{A} \wedge d_{\Lambda}\left(\partial_{A}^{\Lambda} L\right) \omega \\
& \in \varrho\left(\mathcal{S}_{\infty}^{1, n}[F ; Y]\right)
\end{aligned}
$$

is said to be its graded Euler-Lagrange operator. Its kernel yields an Euler-Lagrange equation

$$
\begin{aligned}
\delta L & =0, \\
\mathscr{C}_{A} & =\sum(-1)^{|\Lambda|} \theta^{A} \wedge d_{\Lambda}\left(\partial_{A}^{\Lambda} L\right)=0 .
\end{aligned}
$$

We call a pair $\left(\mathcal{S}_{\infty}^{0, n}[F ; Y], L\right)$ the graded Lagrangian system and $\mathcal{S}_{\infty}^{*}[F ; Y]$ its structure algebra.

The following are corollaries of Theorem 13 [12, 16, 23].

Corollary 14. Any variationally trivial odd Lagrangian is $d_{H^{-}}$ exact.

Corollary 15. Given a graded Lagrangian L, there is the global variational formula

$$
\begin{aligned}
d L & =\delta L-d_{H} \Xi_{L}, \quad \Xi \in \mathcal{S}_{\infty}^{n-1}[F ; Y], \\
\Xi_{L} & =L+\sum_{s=0} \theta_{\nu_{s} \cdots \nu_{1}}^{A} \wedge F_{A}^{\lambda \nu_{s} \cdots \nu_{1}} \omega_{\lambda}, \\
F_{A}^{v_{k} \cdots \nu_{1}} & =\partial_{A}^{\nu_{k} \cdots \nu_{1}} \mathscr{L}-d_{\lambda} F_{A}^{\lambda \nu_{k} \cdots \nu_{1}}+\sigma_{A}^{\nu_{k} \cdots \nu_{1}},
\end{aligned}
$$

$$
k=1,2, \ldots,
$$

where local graded functions $\sigma$ obey relations $\sigma_{A}^{\nu}=0$, $\sigma_{A}^{\left(\nu_{k} \nu_{k-1}\right) \cdots \nu_{1}}=0$.

The form $\Xi_{L}(67)$ provides a global Lepage equivalent of a graded Lagrangian $L$.

Given a graded Lagrangian system $\left(\mathcal{S}_{\infty}^{*}[F ; Y], L\right)$, by its infinitesimal transformations are meant graded derivations of a graded commutative ring $\delta_{\infty}^{0}[F ; Y]$. These derivations constitute a $\delta_{\infty}^{0}[F ; Y]$-module $\mathfrak{D} \delta_{\infty}^{0}[F ; Y]$ which is a real Lie superalgebra with respect to the Lie superbracket (12). The following holds [16]. 
Theorem 16. A derivation module $\mathfrak{d} \mathcal{S}_{\infty}^{0}[F ; Y]$ is isomorphic to the $\mathcal{S}_{\infty}^{0}[F ; Y]$-dual $\mathcal{S}_{\infty}^{1}[F ; Y]^{*}$ of the module of graded oneforms $\mathcal{S}_{\infty}^{1}[F ; Y]$.

In particular, it follows that the DBGA $\mathcal{S}_{\infty}^{*}[F ; Y]$ is minimal differential calculus over a graded commutative ring $\mathcal{S}_{\infty}^{0}[F ; Y]$. Restricted to the coordinate chart $(39)$ of $J^{\infty} Y$, an algebra $\mathcal{S}_{\infty}^{*}[F ; Y]$ is a free $\mathcal{S}_{\infty}^{0}[F ; Y]$-module generated by one-forms $d x^{\lambda}, \theta_{\Lambda}^{A}$.

Due to the isomorphism in Theorem 16, any graded derivation $\vartheta \in \mathfrak{D} \mathcal{S}_{\infty}^{0}[F ; Y]$ takes a form

$$
\vartheta=\vartheta^{\lambda} \partial_{\lambda}+\vartheta^{A} \partial_{A}+\sum_{0<|\Lambda|} \vartheta_{\Lambda}^{A} \partial_{A}^{\Lambda}
$$

Given $\vartheta \in \mathfrak{D} \mathcal{S}_{\infty}^{0}[F ; Y]$ and $\phi \in \mathcal{S}_{\infty}^{1}[F ; Y]$, let $\left.\vartheta\right\rfloor \phi$ denote the corresponding interior product. Extended to the DBGA $\mathcal{S}_{\infty}^{*}[F ; Y]$, it obeys a rule

$$
\begin{array}{r}
\left.\vartheta](\phi \wedge \sigma)=(\vartheta\rfloor \phi) \wedge \sigma+(-1)^{|\phi|+[\phi][\vartheta]} \phi \wedge(\vartheta\rfloor \sigma\right), \\
\phi, \sigma \in \mathcal{S}_{\infty}^{*}[F ; Y] .
\end{array}
$$

Every graded derivation $\vartheta(68)$ of a ring $\mathcal{S}_{\infty}^{0}[F ; Y]$ yields a Lie derivative

$$
\begin{aligned}
\mathbf{L}_{\vartheta} \phi & =\vartheta\rfloor d \phi+d(\vartheta\rfloor \phi), \\
\mathbf{L}_{\vartheta}(\phi \wedge \sigma) & =\mathbf{L}_{\vartheta}(\phi) \wedge \sigma+(-1)^{[\vartheta][\phi]} \phi \wedge \mathbf{L}_{\vartheta}(\sigma),
\end{aligned}
$$

of a DBGA $\delta_{\infty}^{*}[F ; Y]$. The graded derivation $\vartheta(68)$ is called contact if a Lie derivative $\mathbf{L}_{\vartheta}$ preserves the ideal of contact graded forms of $S_{\infty}^{*}[F ; Y]$ generated by contact one-forms.

Lemma 17. With respect to the local generating basis $\left(s^{A}\right)$ for the DBGA $\mathcal{S}_{\infty}^{*}[F ; Y]$, any of its contact graded derivations takes a form

$$
\begin{aligned}
\vartheta & =\vartheta_{H}+\vartheta_{V} \\
& =v^{\lambda} d_{\lambda}+\left[v^{A} \partial_{A}+\sum_{|\Lambda|>0} d_{\Lambda}\left(v^{A}-s_{\mu}^{A} v^{\mu}\right) \partial_{A}^{\Lambda}\right],
\end{aligned}
$$

where $\vartheta_{H}$ and $\vartheta_{V}$ denote the horizontal and vertical parts of $\vartheta$ [16].

A glance at expression (71) shows that a contact graded derivation $\vartheta$ is the infinite order jet prolongation $\vartheta=J^{\infty} v$ of its restriction

$$
v=v^{\lambda} \partial_{\lambda}+v^{A} \partial_{A}=v_{H}+v_{V}=v^{\lambda} d_{\lambda}+\left(u^{A} \partial_{A}-s_{\lambda}^{A} \partial_{A}^{\lambda}\right)
$$

to a graded commutative ring $S^{0}[F ; Y]$. We call $v(72)$ the generalized graded vector field on a graded manifold $\left(Y, \mathfrak{A}_{F}\right)$. This fails to be a graded vector field on $\left(Y, \mathfrak{A}_{F}\right)$ in general because its component may depend on jets of elements of the local generating basis for $\left(Y, \mathfrak{A}_{F}\right)$. reads

In particular, the vertical contact graded derivation (71)

$$
\vartheta=v^{A} \partial_{A}+\sum_{|\Lambda|>0} d_{\Lambda} v^{A} \partial_{A}^{\Lambda}
$$

It is said to be nilpotent if

$$
\begin{aligned}
\mathbf{L}_{\vartheta} & \left(\mathbf{L}_{\vartheta} \phi\right) \\
& =\sum\left(v_{\Sigma}^{B} \partial_{B}^{\Sigma}\left(v_{\Lambda}^{A}\right) \partial_{A}^{\Lambda}+(-1)^{\left[s^{B}\right]\left[v^{A}\right]} v_{\Sigma}^{B} v_{\Lambda}^{A} \partial_{B}^{\Sigma} \partial_{A}^{\Lambda}\right) \phi \\
& =0
\end{aligned}
$$

for any horizontal graded form $\phi \in S_{\infty}^{0, *}$. It is nilpotent only if it is odd and iff the equality

$$
\mathbf{L}_{\vartheta}\left(v^{A}\right)=\sum v_{\Sigma}^{B} \partial_{B}^{\Sigma}\left(v^{A}\right)=0
$$

holds for all $v^{A}[16]$.

Remark 18. If there is no danger of confusion, the common symbol $v$ further stands for a generalized graded vector field $v(72)$, the contact graded derivation $\vartheta$ determined by $v$, and the Lie derivative $\mathbf{L}_{\vartheta}$. We agree to call all these operators, simply, a graded derivation of the structure algebra of a graded Lagrangian system.

Remark 19. For the sake of convenience, right graded derivations $\overleftarrow{v}=\overleftarrow{\partial}_{A} v^{A}$ also are considered. They act on graded functions and differential forms $\phi$ on the right by the rules

$$
\begin{aligned}
\overleftarrow{v}(\phi) & =d \phi[\overleftarrow{v}+d(\phi\lfloor\overleftarrow{v}), \\
\overleftarrow{v}\left(\phi \wedge \phi^{\prime}\right) & =(-1)^{\left[\phi^{\prime}\right]} \overleftarrow{v}(\phi) \wedge \phi^{\prime}+\phi \wedge \overleftarrow{v}\left(\phi^{\prime}\right) .
\end{aligned}
$$

Given a Lagrangian system $\left(\mathcal{S}_{\infty}^{*}[F ; Y], L\right)$, the contact graded derivation $\vartheta(71)$ is called the variational symmetry of a Lagrangian $L$ if a Lie derivative $\mathrm{L}_{\vartheta} L$ of $L$ along $\vartheta$ is $d_{H^{-}}$ exact; that is, $\mathbf{L}_{\vartheta} L=d_{H} \sigma$. Then the following is a corollary of the variational formula (66) [16].

Theorem 20. The Lie derivative of a graded Lagrangian along any contact graded derivation (71) admits the decomposition

$$
\left.\left.\left.\mathbf{L}_{\vartheta} L=v_{V}\right\rfloor \delta L+d_{H}\left(h_{0}(\vartheta\rfloor \Xi_{L}\right)\right)+d_{V}\left(v_{H}\right\rfloor \omega\right) \mathscr{L},
$$

where $\Xi_{L}$ is the Lepage equivalent (67) of a Lagrangian $L$.

A glance at expression (77) shows the following.

Lemma 21. (i) A contact graded derivation $\vartheta$ is a variational symmetry only if it is projected onto $X$. (ii) It is a variational symmetry iff its vertical part $\vartheta_{V}(71)$ is well.

\section{Gauge Symmetries}

Treating gauge symmetries of Lagrangian theory, one usually follows Yang-Mills gauge theory on principal bundles. This notion of gauge symmetries has been generalized to Lagrangian theory on an arbitrary fibre bundle [18]. Here, we extend it to Lagrangian theory on graded bundles.

Let $\left(S_{\infty}^{*}[F ; Y], L\right)$ be a graded Lagrangian system on a graded bundle $\left(X, Y, \mathfrak{A}_{F}\right)$ with the local generating basis $\left(s^{A}\right)$. Let $E=E^{0} \oplus E^{1}$ be a graded vector bundle over $X$ possessing 
an even part $E^{0} \rightarrow X$ and the odd one $E^{1} \rightarrow X$. We regard it as a composite bundle

$$
E \longrightarrow E^{0} \longrightarrow X
$$

and consider a graded bundle $\left(X, E^{0}, \mathfrak{\mathfrak { A }}_{E}\right)$ modelled over it. Then we define product (52) of graded bundles $\left(X, Y, \mathfrak{A}_{F}\right)$ and $\left(X, E^{0}, \mathfrak{A}_{E}\right.$ ) over product (53) of the composite bundles $E$ (78) and $F$ (37). It reads $\left(X, E_{X}^{0} \underset{X}{\times}, \mathfrak{\mathfrak { A }}_{E_{X} \times}\right)$. Let us consider the corresponding DBGA

$$
\mathcal{S}_{\infty}^{*}[\underset{X}{E} \underset{X}{\times} F \underset{X}{0} \underset{Y}{\times}]
$$

together with monomorphisms (55) of DBGAs

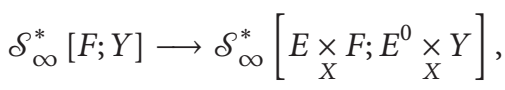

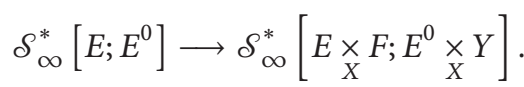
back

Given a Lagrangian $L \in \mathcal{S}_{\infty}^{0, n}[F ; Y]$, let us define its pull-

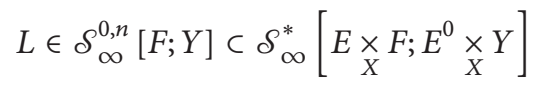

and consider an extended Lagrangian system

$$
\left(\mathcal{S}_{\infty}^{*}\left[\underset{X}{E \times} F ; \underset{X}{0} \times E^{0}\right], L\right)
$$

provided with the local generating basis $\left(s^{A}, c^{r}\right)$.

Definition 22. A gauge transformation of the Lagrangian $L$ (81) is defined to be a contact graded derivation $\vartheta$ of the ring $\mathcal{S}_{\infty}^{0}\left[E \underset{X}{\times} F ; E^{0} \underset{X}{\times} Y\right](79)$ such that a derivation $\vartheta$ equals zero on a subring $\mathcal{S}_{\infty}^{0}\left[E ; E^{0}\right] \subset \mathcal{S}_{\infty}^{0}\left[E \underset{X}{\times} F ; E_{X}^{0} \underset{X}{\times} Y\right] . \mathrm{A}$ gauge transformation $\vartheta$ is called the gauge symmetry if it is a variational symmetry of the Lagrangian $L(81)$.

In view of the first condition in Definition 22, the variables $c^{r}$ of the extended Lagrangian system (82) can be treated as gauge parameters of a gauge symmetry $\vartheta$. Furthermore, we additionally assume that a gauge symmetry $\vartheta$ is linear in gauge parameters $c^{r}$ and their jets $c_{\Lambda}^{r}$ (see Remark 35). Then the generalized graded vector field $v(72)$ reads

$$
\begin{aligned}
v= & \left(\sum_{0 \leq|\Lambda| \leq m} v_{r}^{\lambda \Lambda}\left(x^{\mu}\right) c_{\Lambda}^{r}\right) \partial_{\lambda} \\
& +\left(\sum_{0 \leq|\Lambda| \leq m} v_{r}^{A \Lambda}\left(x^{\mu}, s_{\Sigma}^{B}\right) c_{\Lambda}^{r}\right) \partial_{A} .
\end{aligned}
$$

In accordance with Remark 18, we also call it the gauge symmetry.

By virtue of item (ii) of Lemma 21, the generalized vector field $v(83)$ is a gauge symmetry iff its vertical part is so. Therefore, we can restrict our consideration to vertical gauge symmetries.

\section{Noether and Higher-Stage Noether Identities}

Without loss of generality, let a Lagrangian $L$ be even and its Euler-Lagrange operator $\delta L(64)$ at least of first order. This operator takes its values into a graded vector bundle

$$
\overline{V F}=V^{*} F \bigoplus_{F} \bigwedge^{n} T^{*} X \longrightarrow F,
$$

where $V^{*} F$ is the vertical cotangent bundle of $F \rightarrow X$. It however is not a vector bundle over $Y$. Therefore, we restrict our consideration to a case of the pull-back composite bundle F (37):

$$
F=Y \underset{X}{\times} F^{1} \longrightarrow Y \longrightarrow X
$$

where $F^{1} \rightarrow X$ is a vector bundle.

Remark 23. Let us introduce the following notation. Given the vertical tangent bundle $V E$ of a fibre bundle $E \rightarrow X$, by its density-dual bundle is meant a fibre bundle

$$
\overline{V E}=V^{*} E \bigoplus_{E} \bigwedge^{n} T^{*} X .
$$

If $E \rightarrow X$ is a vector bundle, we have

$$
\overline{V E}=\bar{E} \underset{X}{\times} E, \quad \bar{E}=E^{*} \bigoplus_{X} \bigwedge^{n} T^{*} X,
$$

where $\bar{E}$ is called the density-dual of $E$. Let

$$
E=E^{0} \bigoplus_{X} E^{1}
$$

be a graded vector bundle over $X$. Its graded density-dual is defined to be $\bar{E}=\bar{E}^{1} \oplus \bar{E}^{0}$ with an even part $\bar{E}^{1} \rightarrow X$ and the odd one $\bar{E}^{1} \rightarrow X$. Given the graded vector bundle $E$ (88), we consider a product $\left(X, E^{0} \times \underset{X}{\times}, \mathfrak{\mathcal { A }}_{E \times F}\right)$ of graded bundles over product (53) of the composite bundles $E \rightarrow E^{0} \rightarrow X$ and $F$ (37) and the corresponding DBGA which we denote:

$$
P_{\infty}^{*}[F \underset{X}{\times} E ; Y]=\mathcal{S}_{\infty}^{*}\left[F \underset{X}{\times} E ; Y \underset{X}{\times} E^{0}\right] .
$$

In particular, we treat the composite bundle $F$ (37) as a graded vector bundle over $Y$ possessing only an odd part. The density-dual (86) of the vertical tangent bundle $V F$ of $F \rightarrow X$ is $\overline{V F}$ (84). If $F$ is the pull-back bundle (85), then

$$
\overline{V F}=\bar{F}^{1} \bigoplus_{Y}\left(\left(V^{*} Y \bigoplus_{Y} \bigwedge^{n} T^{*} X\right) \bigoplus_{Y} F^{1}\right)
$$

is a graded vector bundle over $Y$. It can be seen as product (53) of composite bundles

$$
\overline{V F^{1}}=\bar{F}^{1} \bigoplus_{X} F^{1} \longrightarrow \bar{F}^{1} \longrightarrow \mathrm{X}, \quad \overline{V Y} \longrightarrow Y \longrightarrow X,
$$


and we consider the corresponding graded bundle (52) and the DBGA (54) which we denote

$$
\begin{aligned}
\mathscr{P}_{\infty}^{*}[\overline{V F} ; Y] & =\mathcal{S}_{\infty}^{*}\left[\overline{V F} ; \underset{X}{\left.\underset{X}{\times} \bar{F}^{1}\right]}\right. \\
& =\mathcal{S}_{\infty}^{*}\left[\overline{V F}^{1} \underset{X}{\underset{V Y}{\times}} \underset{X}{\times} \underset{X}{\times} \bar{F}^{1}\right] .
\end{aligned}
$$

Lemma 24. One can associate with any graded Lagrangian system $\left(\mathcal{S}_{\infty}^{*}[F ; Y], L\right)$ the chain complex (93) whose oneboundaries vanish on-shell.

Proof. Let us consider the density-dual $\overline{V F}(90)$ of the vertical tangent bundle $V F \rightarrow F$, and let us enlarge an original DBGA $\mathcal{S}_{\infty}^{*}[F ; Y]$ to the DBGA $\mathscr{P}_{\infty}^{*}[\overline{V F} ; Y]$ (92) with the local generating basis $\left(s^{A}, \bar{s}_{A}\right),\left[\bar{s}_{A}\right]=[A]+1$. Following the terminology of Lagrangian BRST theory $[15,19]$, we agree to call its elements $\bar{s}_{A}$ the antifields of antifield number $\operatorname{Ant}\left[\bar{s}_{A}\right]=1$. A DBGA $\mathscr{P}_{\infty}^{*}[\overline{V F} ; Y]$ is endowed with the nilpotent right graded derivation $\bar{\delta}=\overleftarrow{\partial}^{A} \mathscr{E}_{A}$, where $\mathscr{E}_{A}$ are the variational derivatives (64). Then we have a chain complex

$$
0 \longleftarrow \operatorname{Im} \bar{\delta} \stackrel{\bar{\delta}}{\longleftarrow} \mathscr{P}_{\infty}^{0, n}[\overline{V F} ; Y]_{1} \stackrel{\bar{\delta}}{\longleftarrow} \mathscr{P}_{\infty}^{0, n}[\overline{V F} ; Y]_{2}
$$

of graded densities of antifield number $\leq 2$. Its oneboundaries $\bar{\delta} \Phi, \Phi \in \mathscr{P}_{\infty}^{0, n}[\overline{V F} ; Y]_{2}$, by the very definition, vanish on-shell.

Any one-cycle

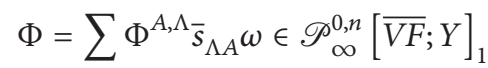

of complex (93) is a differential operator on a bundle $\overline{V F}$ such that it is linear on fibres of $\overline{V F} \rightarrow F$ and its kernel contains the graded Euler-Lagrange operator $\delta L$ (64); that is,

$$
\begin{aligned}
\bar{\delta} \Phi & =0, \\
\sum \Phi^{A, \Lambda} d_{\Lambda} \mathscr{E}_{A} \omega & =0 .
\end{aligned}
$$

Then one can say that one-cycles (94) define the NI (95) of an Euler-Lagrange operator $\delta L$, which we agree to call the NI of a graded Lagrangian system $\left(\mathcal{S}_{\infty}^{*}[F ; Y], L\right)[2]$.

In particular, one-chains $\Phi(94)$ are necessarily NI if they are boundaries. Therefore, these NI are called trivial. They are of the form

$$
\begin{aligned}
\Phi=\sum T^{(A \Lambda)(B \Sigma)} d_{\Sigma} \mathscr{E}_{B} \bar{s}_{\Lambda A} \omega & \\
T^{(A \Lambda)(B \Sigma)} & =-(-1)^{[A][B]} T^{(B \Sigma)(A \Lambda)} .
\end{aligned}
$$

Accordingly, nontrivial NI modulo trivial ones are associated with elements of the first homology $H_{1}(\bar{\delta})$ of complex (93). A Lagrangian $L$ is called degenerate if there are nontrivial NI.

Nontrivial NI can obey first-stage NI. In order to describe them, let us assume that a module $H_{1}(\bar{\delta})$ is finitely generated.
Namely, there exists a graded projective $C^{\infty}(X)$-module $\mathscr{C}_{(0)} \subset H_{1}(\bar{\delta})$ of finite rank possessing a local basis $\left\{\Delta_{r} \omega\right\}$ :

$$
\Delta_{r} \omega=\sum \Delta_{r}^{A, \Lambda} \bar{s}_{\Lambda A} \omega, \quad \Delta_{r}^{A, \Lambda} \in \mathcal{S}_{\infty}^{0}[F ; Y],
$$

such that any element $\Phi \in H_{1}(\bar{\delta})$ factorizes as

$$
\Phi=\sum \Phi^{r, \Xi} d_{\Xi} \Delta_{r} \omega, \quad \Phi^{r, \Xi} \in \mathcal{S}_{\infty}^{0}[F ; Y]
$$

through elements (97) of $\mathscr{C}_{(0)}$. Thus, all nontrivial NI (95) result from the NI

$$
\bar{\delta} \Delta_{r}=\sum \Delta_{r}^{A, \Lambda} d_{\Lambda} \mathscr{E}_{A}=0,
$$

called the complete NI. Note that factorization (98) is independent of specification of a local basis $\left\{\Delta_{r} \omega\right\}$ and, being representatives of $H_{1}(\bar{\delta})$, graded densities $\Delta_{r} \omega(97)$ are not $\bar{\delta}$-exact.

A Lagrangian system whose nontrivial NI are finitely generated is called finitely degenerate. Hereafter, degenerate Lagrangian systems only of this type are considered.

Lemma 25. If the homology $H_{1}(\bar{\delta})$ of complex (93) is finitely generated in the above-mentioned sense, this complex can be extended to the one-exact chain complex (102) with a boundary operator whose nilpotency conditions are equivalent to the complete NI (99).

Proof. By virtue of Theorem 5, a graded module $\mathscr{C}_{(0)}$ is isomorphic to that of sections of the density-dual $\bar{E}_{0}$ of some graded vector bundle $E_{0} \rightarrow X$. Let us enlarge $\mathscr{P}_{\infty}^{*}[\overline{V F} ; Y]$ to a DBGA

$$
\begin{aligned}
\overline{\mathscr{P}}_{\infty}^{*}\{0\} & =\mathscr{P}_{\infty}^{*}\left[\overline{V F} \underset{X}{\times} \bar{E}_{0} ; Y\right] \\
& =\mathcal{S}_{\infty}^{*}\left[\overline{V F} \underset{X}{\times} \bar{E}_{0} ; \bar{E}_{0}^{1} \underset{X}{\times} \bar{F}^{1} \underset{X}{\times}\right]
\end{aligned}
$$

with a local generating basis $\left(s^{A}, \bar{s}_{A}, \bar{c}_{r}\right)$ where $\bar{c}_{r}$ are antifields of Grassmann parity $\left[\bar{c}_{r}\right]=\left[\Delta_{r}\right]+1$ and antifield number $\operatorname{Ant}\left[\bar{c}_{r}\right]=2$. DBGA (100) admits an odd right graded derivation

$$
\delta_{0}=\bar{\delta}+\overleftarrow{\partial}^{r} \Delta_{r}
$$

which is nilpotent iff the complete NI (99) hold. Then $\delta_{0}(101)$ is a boundary operator of a chain complex

$$
\begin{aligned}
& 0 \longleftarrow \operatorname{Im} \bar{\delta} \stackrel{\bar{\delta}}{\longleftarrow} \mathscr{P}_{\infty}^{0, n}[\overline{V F} ; Y]_{1} \stackrel{\delta_{0}}{\longleftarrow} \overline{\mathscr{P}}_{\infty}^{0, n}\{0\}_{2} \\
& \stackrel{\delta_{0}}{\longleftarrow} \overline{\mathscr{P}}_{\infty}^{0, n}\{0\}_{3}
\end{aligned}
$$

of graded densities of antifield number $\leq 3$. Let $H_{*}\left(\delta_{0}\right)$ denote its homology. We have $H_{0}\left(\delta_{0}\right)=H_{0}(\bar{\delta})=0$. Furthermore, any one-cycle $\Phi$ up to a boundary takes the form (98) and, therefore, it is a $\delta_{0}$-boundary

$$
\Phi=\sum \Phi^{r, \Xi} d_{\Xi} \Delta_{r} \omega=\delta_{0}\left(\sum \Phi^{r, \Xi} \bar{c}_{\Xi r} \omega\right) .
$$

Hence, $H_{1}\left(\delta_{0}\right)=0$; that is, complex (102) is one-exact. 
Let us consider the second homology $\mathrm{H}_{2}\left(\delta_{0}\right)$ of complex (102). Its two-chains read

$$
\Phi=G+H=\sum G^{r, \Lambda} \bar{c}_{\Lambda r} \omega+\sum H^{(A, \Lambda)(B, \Sigma)} \bar{s}_{\Lambda A} \bar{s}_{\Sigma B} \omega .
$$

Its two-cycles define the first-stage NI

$$
\begin{aligned}
\delta_{0} \Phi & =0, \\
\sum G^{r, \Lambda} d_{\Lambda} \Delta_{r} \omega & =-\bar{\delta} H .
\end{aligned}
$$

Conversely, let equality (105) hold. Then it is a cycle condition of the two-chain (104).

Note that this definition of first-stage NI is independent of specification of a generating module $\mathscr{C}_{(0)}$ up to chain isomorphisms between complexes (102).

The first-stage NI (105) are trivial either if the two-cycle $\Phi$ (104) is a $\delta_{0}$-boundary or its summand $G$ vanishes on-shell. Therefore, nontrivial first-stage NI fails to exhaust the second homology $\mathrm{H}_{2}\left(\delta_{0}\right)$ of complex (102) in general.

Lemma 26. Nontrivial first-stage NI modulo trivial ones are identified with elements of the homology $\mathrm{H}_{2}\left(\delta_{0}\right)$ iff any $\bar{\delta}$-cycle $\phi \in \overline{\mathscr{P}}_{\infty}^{0, n}\{0\}_{2}$ is a $\delta_{0}$-boundary.

Proof. It suffices to show that if a summand $G$ of a two-cycle $\Phi(104)$ is $\bar{\delta}$-exact, then $\Phi$ is a boundary. If $G=\bar{\delta} \Psi$, let us write

$$
\Phi=\delta_{0} \Psi+\left(\bar{\delta}-\delta_{0}\right) \Psi+H
$$

Hence, cycle condition (105) reads

$$
\delta_{0} \Phi=\bar{\delta}\left(\left(\bar{\delta}-\delta_{0}\right) \Psi+H\right)=0 .
$$

Since any $\bar{\delta}$-cycle $\phi \in \overline{\mathscr{P}}_{\infty}^{0, n}\{0\}_{2}$, by assumption, is $\delta_{0}$-exact, then $\left(\bar{\delta}-\delta_{0}\right) \Psi+H$ is a $\delta_{0}$-boundary. Consequently, $\Phi(106)$ is $\delta_{0}$-exact. Conversely, let $\Phi \in \overline{\mathscr{P}}_{\infty}^{0, n}\{0\}_{2}$ be a $\bar{\delta}$-cycle; that is,

$$
\begin{aligned}
\bar{\delta} \Phi & =2 \Phi^{(A, \Lambda)(B, \Sigma)} \bar{s}_{\Lambda A} \bar{\delta}_{\bar{s}_{\Sigma B} \omega} \\
& =2 \Phi^{(A, \Lambda)(B, \Sigma)} \bar{s}_{\Lambda A} d_{\Sigma} \mathscr{E}_{B} \omega=0 .
\end{aligned}
$$

It follows that $\Phi^{(A, \Lambda)(B, \Sigma)} \bar{\delta} \bar{s}_{\Sigma B}=0$ for all indices $(A, \Lambda)$. Omitting a $\bar{\delta}$-boundary term, we obtain

$$
\Phi^{(A, \Lambda)(B, \Sigma)} \bar{s}_{\Sigma B}=G^{(A, \Lambda)(r, \Xi)} d_{\Xi} \Delta_{r} .
$$

Hence, $\Phi$ takes a form

$$
\Phi=G^{\prime(A, \Lambda)(r, \Xi)} d_{\Xi} \Delta_{r} \bar{s}_{\Lambda A} \omega .
$$

Then there exists a three-chain $\Psi=G^{\prime(A, \Lambda)(r, \Xi)} \overline{\mathcal{c}}_{\Xi r} \bar{s}_{\Lambda A} \omega$ such that

$$
\delta_{0} \Psi=\Phi+\sigma=\Phi+G^{\prime \prime(A, \Lambda)(r, \Xi)} d_{\Lambda} \mathscr{E}_{A} \bar{c}_{\Xi r} \omega
$$

Owing to the equality $\bar{\delta} \Phi=0$, we have $\delta_{0} \sigma=0$. Thus, $\sigma$ in expression (111) is a $\bar{\delta}$-exact $\delta_{0}$-cycle. By assumption, it is $\delta_{0^{-}}$ exact; that is, $\sigma=\delta_{0} \psi$. Thus, a $\bar{\delta}$-cycle $\Phi$ is a $\delta_{0}$-boundary.
A degenerate Lagrangian system is called reducible if it admits nontrivial first-stage NI.

If the condition of Lemma 26 is satisfied, let us assume that nontrivial first-stage NI are finitely generated as follows. There exists a graded projective $C^{\infty}(X)$-module $\mathscr{C}_{(1)} \subset$ $H_{2}\left(\delta_{0}\right)$ of finite rank possessing a local basis $\left\{\Delta_{r_{1}} \omega\right\}$ :

$$
\Delta_{r_{1}} \omega=\sum \Delta_{r_{1}}^{r, \Lambda} \bar{c}_{\Lambda r} \omega+h_{r_{1}} \omega
$$

such that any element $\Phi \in H_{2}\left(\delta_{0}\right)$ factorizes as

$$
\Phi=\sum \Phi^{r_{1}, \Xi} d_{\Xi} \Delta_{r_{1}} \omega, \quad \Phi^{r_{1}, \Xi} \in \mathcal{S}_{\infty}^{0}[F ; Y]
$$

through elements (112) of $\mathscr{C}_{(1)}$. Thus, all nontrivial first-stage NI (105) result from the equalities

$$
\sum \Delta_{r_{1}}^{r, \Lambda} d_{\Lambda} \Delta_{r}+\bar{\delta} h_{r_{1}}=0
$$

called the complete first-stage NI. Note that, by virtue of the condition of Lemma 26, the first summands of the graded densities $\Delta_{r_{1}} \omega(112)$ are not $\bar{\delta}$-exact. A degenerate Lagrangian system is called finitely reducible if it admits finitely generated nontrivial first-stage NI.

Lemma 27. The one-exact complex (102) of a finitely reducible Lagrangian system is extended to the two-exact one (117) with a boundary operator whose nilpotency conditions are equivalent to the complete NI (99) and the complete first-stage NI (114).

Proof. By virtue of Theorem 5, a graded module $\mathscr{C}_{(1)}$ is isomorphic to that of sections of the density-dual $\bar{E}_{1}$ of some graded vector bundle $E_{1} \rightarrow X$. Let us enlarge the DBGA $\overline{\mathscr{P}}_{\infty}^{*}\{0\}(100)$ to a DBGA

$$
\overline{\mathscr{P}}_{\infty}^{*}\{1\}=\mathscr{P}_{\infty}^{*}\left[\overline{V F} \underset{X}{\times} \bar{E}_{0} \underset{X}{\times} \bar{E}_{1} ; Y\right]
$$

with a local basis $\left\{s^{A}, \bar{s}_{A}, \bar{c}_{r}, \bar{c}_{r_{1}}\right\}$ where $\bar{c}_{r_{1}}$ are first-stage antifields of Grassmann parity $\left[\bar{c}_{r_{1}}\right]=\left[\Delta_{r_{1}}\right]+1$ and antifield number 3 . This DBGA is provided with the odd right graded derivation

$$
\delta_{1}=\delta_{0}+\stackrel{\llcorner}{\partial}^{r_{1}} \Delta_{r_{1}}
$$

which is nilpotent iff the complete NI (99) and the complete first-stage NI (114) hold. Then $\delta_{1}(116)$ is a boundary operator of a chain complex

$$
\begin{gathered}
0 \longleftarrow \operatorname{Im} \bar{\delta} \stackrel{\bar{\delta}}{\longleftarrow} \mathscr{P}_{\infty}^{0, n}[\overline{V F} ; Y]_{1} \stackrel{\delta_{0}}{\longleftarrow} \overline{\mathscr{P}}_{\infty}^{0, n}\{0\}_{2} \\
\stackrel{\delta_{1}}{\longleftarrow} \overline{\mathscr{P}}_{\infty}^{0, n}\{1\}_{3} \stackrel{\delta_{1}}{\longleftarrow} \overline{\mathscr{P}}_{\infty}^{0, n}\{1\}_{4}
\end{gathered}
$$

of graded densities of antifield number $\leq 4$. Let $H_{*}\left(\delta_{1}\right)$ denote its homology. We have

$$
\begin{aligned}
& H_{0}\left(\delta_{1}\right)=H_{0}(\bar{\delta}), \\
& H_{1}\left(\delta_{1}\right)=H_{1}\left(\delta_{0}\right)=0 .
\end{aligned}
$$


By virtue of expression (113), any two-cycle of the complex (117) is a boundary

$$
\Phi=\sum \Phi^{r_{1}, \Xi} d_{\Xi} \Delta_{r_{1}} \omega=\delta_{1}\left(\sum \Phi^{r_{1}, \Xi} \bar{c}_{\Xi r_{1}} \omega\right)
$$

It follows that $H_{2}\left(\delta_{1}\right)=0$; that is, complex (117) is two-exact.

If the third homology $H_{3}\left(\delta_{1}\right)$ of complex (117) is not trivial, its elements correspond to second-stage NI which the complete first-stage ones satisfy, and so on. Iterating the arguments, we say that a degenerate graded Lagrangian system $\left(\mathcal{S}_{\infty}^{*}[F ; Y], L\right)$ is $N$-stage reducible if it admits finitely generated nontrivial $N$-stage NI, but no nontrivial $(N+1)$ stage ones. It is characterized as follows [2]:

(i) There are graded vector bundles $E_{0}, \ldots, E_{N}$ over $X$, and a DBGA $\mathscr{P}_{\infty}^{*}[\overline{V F} ; Y]$ is enlarged to a DBGA

$\overline{\mathscr{P}}_{\infty}^{*}\{N\}=\mathscr{P}_{\infty}^{*}\left[\overline{V F} \underset{X}{\times} \bar{E}_{0} \underset{X}{\times} \cdots \underset{X}{\times} \bar{E}_{N} ; Y\right]$

with a local generating basis $\left(s^{A}, \bar{s}_{A}, \bar{c}_{r}, \bar{c}_{r_{1}}, \ldots, \bar{c}_{r_{N}}\right)$ where $\bar{c}_{r_{k}}$ are $k$-stage antifields of antifield number Ant $\left[\bar{c}_{r_{k}}\right]=k+2$.

(ii) DBGA (120) is provided with a nilpotent right graded derivation

$$
\begin{aligned}
\delta_{\mathrm{KT}}= & \delta_{N}=\bar{\delta}+\sum \breve{\partial}^{r} \Delta_{r}^{A, \Lambda} \bar{s}_{\Lambda A}+\sum_{1 \leq k \leq N} \breve{\partial}^{r_{k}} \Delta_{r_{k}}, \\
\Delta_{r_{k}} \omega= & \sum \Delta_{r_{k}}^{r_{k-1}, \Lambda} \bar{c}_{\Lambda r_{k-1}} \omega \\
& +\sum\left(h_{r_{k},\left(r_{k-2}, \Sigma\right)(A, \Xi)} \bar{c}_{\Sigma r_{k-2}} \bar{s}_{\Xi A}+\cdots\right) \omega \\
\in & \overline{\mathscr{P}}_{\infty}^{0, n}\{k-1\}_{k+1},
\end{aligned}
$$

of antifield number -1 . The index $k=-1$ here stands for $\bar{s}_{A}$. The nilpotent derivation $\delta_{\mathrm{KT}}(121)$ is called the KT operator.

(iii) With this graded derivation, a module $\overline{\mathscr{P}}_{\infty}^{0, n}\{N\}_{\leq N+3}$ of densities of antifield number $\leq(N+3)$ is decomposed into the exact KT chain complex

$$
\begin{aligned}
0 \longleftarrow & \operatorname{Im} \bar{\delta} \stackrel{\bar{\delta}}{\longleftarrow} \mathscr{P}_{\infty}^{0, n}[\overline{V F} ; Y]_{1} \stackrel{\delta_{0}}{\longleftarrow} \overline{\mathscr{P}}_{\infty}^{0, n}\{0\}_{2} \\
& \stackrel{\delta_{1}}{\longleftarrow} \overline{\mathscr{P}}_{\infty}^{0, n}\{1\}_{3} \ldots \stackrel{\delta_{N-1}}{\longleftarrow} \overline{\mathscr{P}}_{\infty}^{0, n}\{N-1\}_{N+1} \\
& \stackrel{\delta_{\mathrm{KT}}}{\longleftarrow} \overline{\mathscr{P}}_{\infty}^{0, n}\{N\}_{N+2} \stackrel{\delta_{\mathrm{KT}}}{\longleftarrow} \overline{\mathscr{P}}_{\infty}^{0, n}\{N\}_{N+3}
\end{aligned}
$$

which satisfies the following homology regularity condition.

Condition 1. Any $\delta_{k<N}$-cycle $\phi \in \overline{\mathscr{P}}_{\infty}^{0, n}\{k\}_{k+3} \subset \overline{\mathscr{P}}_{\infty}^{0, n}\{k+1\}_{k+3}$ is a $\delta_{k+1}$-boundary. (iv) The nilpotentness $\delta_{\mathrm{KT}}^{2}=0$ of the KT operator (121) is equivalent to the complete nontrivial NI (99) and the complete nontrivial $(k \leq N)$-stage NI

$$
\begin{aligned}
& \sum \Delta_{r_{k}}^{r_{k-1}, \Lambda} d_{\Lambda}\left(\sum \Delta_{r_{k-1}}^{r_{k-2}, \Sigma} \bar{c}_{\Sigma r_{k-2}}\right) \\
& =-\bar{\delta}\left(\sum h_{r_{k}}^{\left(r_{k-2}, \Sigma\right)(A, \Xi)} \bar{c}_{\Sigma r_{k-2}} \bar{s}_{\Xi A}\right) .
\end{aligned}
$$

This item means the following.

Lemma 28. The $\delta_{k}$-cocycles $\Phi \in \mathscr{P}_{\infty}^{0, n}\{k\}_{k+2}$ are $k$-stage NI, and vice versa.

Proof. Any $(k+2)$-chain $\Phi \in \mathscr{P}_{\infty}^{0, n}\{k\}_{k+2}$ takes a form

$$
\begin{aligned}
\Phi= & G+H \\
= & \sum G^{r_{k}, \Lambda} \bar{c}_{\Lambda r_{k}} \omega \\
& +\sum\left(H^{(A, \Xi)\left(r_{k-1}, \Sigma\right)} \bar{s}_{\Xi A} \bar{c}_{\Sigma r_{k-1}}+\cdots\right) \omega .
\end{aligned}
$$

If it is a $\delta_{k}$-cycle, then

$$
\begin{aligned}
& \sum G^{r_{k}, \Lambda} d_{\Lambda}\left(\sum \Delta_{r_{k}}^{r_{k-1}, \Sigma} \bar{c}_{\Sigma r_{k-1}}\right) \\
& +\bar{\delta}\left(\sum H^{(A, \Xi)\left(r_{k-1}, \Sigma\right)} \bar{s}_{\Xi A} \bar{c}_{\Sigma r_{k-1}}\right)=0
\end{aligned}
$$

are the $k$-stage NI. Conversely, let condition (126) hold. It can be extended to a cycle condition as follows. It is brought into the form

$$
\begin{aligned}
\delta_{k} & \left(\sum G^{r_{k}, \Lambda} \bar{c}_{\Lambda r_{k}}+\sum H^{(A, \Xi)\left(r_{k-1}, \Sigma\right)} \bar{s}_{\Xi A} \bar{c}_{\Sigma r_{k-1}}\right) \\
& =-\sum G^{r_{k}, \Lambda} d_{\Lambda} h_{r_{k}}+\sum H^{(A, \Xi)\left(r_{k-1}, \Sigma\right)} \bar{s}_{\Xi A} d_{\Sigma} \Delta_{r_{k-1}} .
\end{aligned}
$$

A glance at expression (122) shows that the term in its righthand side belongs to $\mathscr{P}_{\infty}^{0, n}\{k-2\}_{k+1}$. It is a $\delta_{k-2}$-cycle and, consequently, a $\delta_{k-1}$-boundary $\delta_{k-1} \Psi$ in accordance with Condition 1 . Then equality (126) is a $\overline{\mathrm{c}}_{\Sigma r_{k-1}}$-dependent part of a cycle condition

$$
\delta_{k}\left(\sum G^{r_{k}, \Lambda} \bar{c}_{\Lambda r_{k}}+\sum H^{(A, \Xi)\left(r_{k-1}, \Sigma\right)} \bar{s}_{\Xi A} \bar{c}_{\Sigma r_{k-1}}-\Psi\right)=0,
$$

but $\delta_{k} \Psi$ does not make a contribution to this condition.

Lemma 29. Trivial $k$-stage $N I$ are $\delta_{k}$-boundaries $\Phi \in$ $\mathscr{P}_{\infty}^{0, n}\{k\}_{k+2}$.

Proof. The $k$-stage NI (126) are trivial either if a $\delta_{k}$-cycle $\Phi$ (125) is a $\delta_{k}$-boundary or its summand $G$ vanishes on-shell. Let us show that if the summand $G$ of $\Phi(125)$ is $\bar{\delta}$-exact, then $\Phi$ is a $\delta_{k}$-boundary. If $G=\bar{\delta} \Psi$, one can write

$$
\Phi=\delta_{k} \Psi+\left(\bar{\delta}-\delta_{k}\right) \Psi+H .
$$

Hence, the $\delta_{k}$-cycle condition reads

$$
\delta_{k} \Phi=\delta_{k-1}\left(\left(\bar{\delta}-\delta_{k}\right) \Psi+H\right)=0 .
$$

By virtue of Condition 1 , any $\delta_{k-1}$-cycle $\phi \in \overline{\mathscr{P}}_{\infty}^{0, n}\{k-1\}_{k+2}$ is $\delta_{k}$-exact. Then $\left(\bar{\delta}-\delta_{k}\right) \Psi+H$ is a $\delta_{k}$-boundary. Consequently, $\Phi(125)$ is $\delta_{k}$-exact. 
Lemma 30. All nontrivial $k$-stage NI (126), by assumption, factorize as

$$
\begin{aligned}
\Phi & =\sum \Phi^{r_{k}, \Xi} d_{\Xi} \Delta_{r_{k}} \omega, \\
\Phi^{r_{1}, \Xi} & \in \mathcal{S}_{\infty}^{0}[F ; Y],
\end{aligned}
$$

through the complete ones (124).

It may happen that a graded Lagrangian system possesses nontrivial NI of any stage. However, we restrict our consideration to $N$-reducible Lagrangians for a finite integer $N$. In this case, the KT operator (121) and the gauge operator (139) contain finite terms.

\section{Second Noether Theorems}

Different variants of the second Noether theorem have been suggested in order to relate reducible NI and gauge symmetries $[2,15,26]$. The inverse second Noether theorem (Theorem 33), which we formulate in homology terms, associates with the KT complex (123) of nontrivial NI the cochain sequence (138) with the ascent operator $\mathbf{u}$ (139) whose components are gauge and higher-stage gauge symmetries of a Lagrangian system. Let us start with the following notation.

Remark 31. Given the DBGA $\overline{\mathscr{P}}_{\infty}^{*}\{N\}(120)$, we consider a DBGA

$$
P_{\infty}^{*}\{N\}=P_{\infty}^{*}\left[F \underset{X}{\underset{X}{x}} E_{0} \underset{X}{\times} \underset{X}{\times} E_{N} ; Y\right],
$$

possessing the local generating basis $\left(s^{A}, c^{r}, c^{r_{1}}, \ldots, c^{r_{N}}\right)$, $\left[c^{r_{k}}\right]=\left[\bar{c}_{r_{k}}\right]+1$, and a DBGA

$$
\begin{aligned}
\mathscr{P}_{\infty}^{*} & \{N\} \\
& =\mathscr{P}_{\infty}^{*}\left[\overline{V F} \underset{X}{\times} E_{0} \underset{X}{\times} \cdots \underset{X}{\times} E_{N} \underset{X}{\times} \bar{E}_{0} \underset{X}{\times} \cdots \underset{X}{\times} \bar{E}_{N} ; Y\right]
\end{aligned}
$$

with a local generating basis $\left(s^{A}, \bar{s}_{A}, c^{r}, c^{r_{1}}, \ldots, c^{r_{N}}, \bar{c}_{r}, \bar{c}_{r_{1}}, \ldots\right.$, $\bar{c}_{r_{N}}$ ). Their elements $c^{r_{k}}$ are called $k$-stage ghosts of ghost number $\operatorname{gh}\left[c^{r_{k}}\right]=k+1$ and antifield number $\operatorname{Ant}\left[c^{r_{k}}\right]=$ $-(k+1)$. A $C^{\infty}(X)$-module $\mathscr{C}^{(k)}$ of $k$-stage ghosts is the density-dual of a module $\mathscr{C}_{(k+1)}$ of $(k+1)$-stage antifields. In accordance with Remark 11, the DBGAs $\overline{\mathscr{P}}_{\infty}^{*}\{N\}(120)$ and the BGDA $P^{*}(N)(132)$ are subalgebras of the DBGA $\mathscr{P}_{\infty}^{*}\{N\}$ (133). The KT operator $\delta_{\mathrm{KT}}$ (121) naturally is extended to a graded derivation of a DBGA $\mathscr{P}_{\infty}^{*}\{N\}$.

Remark 32. Any graded differential form $\phi \in \mathcal{S}_{\infty}^{*}[F ; Y]$ and any finite tuple $\left(f^{\Lambda}\right), 0 \leq|\Lambda| \leq k$, of local graded functions $f^{\Lambda} \in \mathcal{S}_{\infty}^{0}[F ; Y]$ obey the following relations:

$$
\begin{aligned}
& \sum_{0 \leq|\Lambda| \leq k} f^{\Lambda} d_{\Lambda} \phi \wedge \omega \\
& \quad=\sum(-1)^{|\Lambda|} d_{\Lambda}\left(f^{\Lambda}\right) \phi \wedge \omega+d_{H} \sigma,
\end{aligned}
$$

$$
\begin{aligned}
& \sum_{0 \leq \leq \Lambda \mid \leq k}(-1)^{|\Lambda|} d_{\Lambda}\left(f^{\Lambda} \phi\right)=\sum_{0 \leq|\Lambda| \leq k} \eta(f)^{\Lambda} d_{\Lambda} \phi, \\
& \eta(f)^{\Lambda}=\sum_{0 \leq|\Sigma| \leq k-|\Lambda|}(-1)^{|\Sigma+\Lambda|} \frac{(|\Sigma+\Lambda|) !}{|\Sigma| !|\Lambda| !} d_{\Sigma} f^{\Sigma+\Lambda}, \\
& \eta(\eta(f))^{\Lambda}=f^{\Lambda} .
\end{aligned}
$$

Theorem 33. Given the KT complex (123), a module of graded densities $P_{\infty}^{0, n}\{N\}$ is decomposed into a cochain sequence

$$
\begin{aligned}
0 & \longrightarrow \mathcal{S}_{\infty}^{0, n}[F ; Y] \stackrel{\mathbf{u}}{\longrightarrow} P_{\infty}^{0, n}\{N\}^{1} \stackrel{\mathbf{u}}{\longrightarrow} P_{\infty}^{0, n}\{N\}^{2} \\
& \stackrel{\mathbf{u}}{\longrightarrow} \cdots, \\
\mathbf{u} & =u+u^{(1)}+\cdots+u^{(N)} \\
& =u^{A} \frac{\partial}{\partial s^{A}}+u^{r} \frac{\partial}{\partial c^{r}}+\cdots+u^{r} \frac{\partial}{\partial c^{r}},
\end{aligned}
$$

graded in ghost number. Its ascent operator $\mathbf{u}$ (139) is an odd graded derivation of ghost number 1 where $u$ (144) is a variational symmetry of a graded Lagrangian $L$ and the graded derivations $u_{(k)}(149), k=1, \ldots, N$, obey relations (148).

Proof. Given the KT operator (121), let us extend an original Lagrangian $L$ to a Lagrangian

$$
\begin{aligned}
L_{e} & =L+L_{1}=L+\sum_{0 \leq k \leq N} c^{r_{k}} \Delta_{r_{k}} \omega \\
& =L+\delta_{\mathrm{KT}}\left(\sum_{0 \leq k \leq N} c^{r_{k}} \bar{c}_{r_{k}} \omega\right)
\end{aligned}
$$

of zero antifield number. It is readily observed that the KT operator $\delta_{\mathrm{KT}}$ is an exact symmetry of the extended Lagrangian $L_{e} \in \mathscr{P}_{\infty}^{0, n}\{N\}$ (140). Since the graded derivation $\delta_{\mathrm{KT}}$ is vertical, it follows from decomposition (77) that

$$
\begin{aligned}
& {\left[\frac{\bar{\delta}_{\mathscr{L}_{e}}}{\delta \bar{s}_{A}} \mathscr{E}_{A}+\sum_{0 \leq k \leq N} \frac{\bar{\delta} \mathscr{L}_{e}}{\delta \bar{c}_{r_{k}}} \Delta_{r_{k}}\right] \omega} \\
& =\left[v^{A} \mathscr{C}_{A}+\sum_{0 \leq k \leq N} v^{r_{k}} \frac{\delta \mathscr{L}_{e}}{\delta c^{r_{k}}}\right] \omega=d_{H} \sigma, \\
& v^{A}=\frac{\overleftarrow{\delta} \mathscr{L}_{e}}{\delta \bar{s}_{A}}=u^{A}+w^{A} \\
& =\sum c_{\Lambda}^{r} \eta\left(\Delta_{r}^{A}\right)^{\Lambda}+\sum_{1 \leq i \leq N} \sum c_{\Lambda}^{r_{i}} \eta\left(\check{\partial}^{A}\left(h_{r_{i}}\right)\right)^{\Lambda}, \\
& v^{r_{k}}=\frac{\overleftarrow{\delta} \mathscr{L}_{e}}{\delta \bar{c}_{r_{k}}}=u^{r_{k}}+w^{r_{k}} \\
& =\sum c_{\Lambda}^{r_{k+1}} \eta\left(\Delta_{r_{k+1}}^{r_{k}}\right)^{\Lambda}+\sum_{k+1<i \leq N} \sum c_{\Lambda}^{r_{i}} \eta\left(\check{\partial}^{r_{k}}\left(h_{r_{i}}\right)\right)^{\Lambda} .
\end{aligned}
$$


Equality (141) is split into a set of equalities

$$
\begin{aligned}
& \frac{\overleftarrow{\delta}\left(c^{r} \Delta_{r}\right)}{\delta \bar{s}_{A}} \mathscr{E}_{A} \omega=u^{A} \mathscr{E}_{A} \omega=d_{H} \sigma_{0}, \\
& {\left[\frac{\overleftarrow{\delta}\left(c^{r_{k}} \Delta_{r_{k}}\right)}{\delta \bar{s}_{A}} \mathscr{E}_{A}+\sum_{0 \leq i<k} \frac{\overleftarrow{\delta}\left(c^{r_{k}} \Delta_{r_{k}}\right)}{\delta \bar{c}_{r_{i}}} \Delta_{r_{i}}\right] \omega=d_{H} \sigma_{k},}
\end{aligned}
$$

where $k=1, \ldots, N$. A glance at equality (142) shows that, by virtue of decomposition (77), the odd graded derivation

$$
u=u^{A} \partial_{A}, \quad u^{A}=\sum c_{\Lambda}^{r} \eta\left(\Delta_{r}^{A}\right)^{\Lambda}
$$

of $P_{\infty}^{0}\{0\}$ is a variational symmetry of a graded Lagrangian $L$. Every equality (143) falls into a set of equalities graded by the polynomial degree in antifields. Let us consider the equalities which are linear in antifields $\bar{c}_{r_{k-2}}$. We have

$$
\begin{aligned}
& \frac{\overleftarrow{\delta}}{\delta \bar{s}_{A}}\left(c^{r_{k}} \sum h_{r_{k}}^{\left(r_{k-2}, \Sigma\right)(A, \Xi)} \bar{c}_{\Sigma r_{k-2}} \bar{s}_{\Xi A}\right) \mathscr{E}_{A} \omega \\
& \quad+\frac{\overleftarrow{\delta}}{\delta \bar{c}_{r_{k-1}}}\left(c^{r_{k}} \sum \Delta_{r_{k}}^{r_{k-1}^{\prime}, \Sigma} \bar{c}_{\Sigma r_{k-1}^{\prime}}\right) \sum \Delta_{r_{k-1}}^{r_{k-2}, \Xi} \bar{c}_{\Xi r_{k-2}} \omega \\
& =d_{H} \sigma_{k} .
\end{aligned}
$$

This equality is brought into the form

$$
\begin{gathered}
\sum(-1)^{|\Xi|} d_{\Xi}\left(c^{r_{k}} \sum h_{r_{k}}^{\left(r_{k-2}, \Sigma\right)(A, \Xi)} \bar{c}_{\Sigma r_{k-2}}\right) \mathscr{E}_{A} \omega \\
+u^{r_{k-1}} \sum \Delta_{r_{k-1}}^{r_{k-2}, \Xi} \bar{c}_{\Xi r_{k-2}} \omega=d_{H} \sigma_{k} .
\end{gathered}
$$

Using relation (134), we obtain the equality

$$
\begin{aligned}
& \sum c^{r_{k}} h_{r_{k}}^{\left(r_{k-2}, \Sigma\right)(A, \Xi)} \bar{c}_{\Sigma r_{k-2}} d_{\Xi} \mathscr{E}_{A} \omega \\
& \quad+u^{r_{k-1}} \sum \Delta_{r_{k-1}}^{r_{k-2}, \Xi} \bar{c}_{\Xi r_{k-2}} \omega=d_{H} \sigma_{k}^{\prime} .
\end{aligned}
$$

The variational derivative of both of its sides with respect to $\bar{c}_{r_{k-2}}$ leads to the relation

$$
\begin{aligned}
& \sum d_{\Sigma} u^{r_{k-1}} \frac{\partial}{\partial c_{\Sigma}^{r_{k-1}}} u^{r_{k-2}}=\bar{\delta}\left(\alpha^{r_{k-2}}\right), \\
& \alpha^{r_{k-2}}=-\sum \eta\left(h_{r_{k}}^{\left(r_{k-2}\right)(A, \Xi)}\right)^{\Sigma} d_{\Sigma}\left(c^{r_{k}} \bar{s}_{\Xi A}\right),
\end{aligned}
$$

which the odd graded derivation

$$
\begin{aligned}
u^{(k)}=u^{r_{k-1}} \frac{\partial}{\partial c^{r_{k-1}}}=\sum c_{\Lambda}^{r_{k}} \eta\left(\Delta_{r_{k}}^{r_{k-1}}\right)^{\Lambda} \frac{\partial}{\partial c^{r_{k-1}}} & \\
k & =1, \ldots, N,
\end{aligned}
$$

satisfies. Graded derivations $u(144)$ and $u^{(k)}(149)$ constitute the ascent operator (139).

A glance at the variational symmetry $u$ (144) shows that it is a derivation of a ring $P_{\infty}^{0}[0]$ which satisfies Definition 22 . Consequently, $u(144)$ is a gauge symmetry of a graded
Lagrangian $L$ associated with the complete nontrivial NI (99). Therefore, it is a nontrivial gauge symmetry.

Turn now to relation (148). For $k=1$, it takes a form

$$
\sum d_{\Sigma} u^{r} \partial_{r}^{\Sigma} u^{A}=\bar{\delta}\left(\alpha^{A}\right)
$$

of a first-stage gauge symmetry condition on-shell which the nontrivial gauge symmetry $u$ (144) satisfies. Therefore, one can treat the odd graded derivation

$$
u^{(1)}=u^{r} \partial_{r}, \quad u^{r}=\sum c_{\Lambda}^{r_{1}} \eta\left(\Delta_{r_{1}}^{r}\right)^{\Lambda}
$$

as a first-stage gauge symmetry associated with the complete first-stage NI

$$
\sum \Delta_{r_{1}}^{r, \Lambda} d_{\Lambda}\left(\sum \Delta_{r}^{A, \Sigma} \bar{s}_{\Sigma A}\right)=-\bar{\delta}\left(\sum h_{r_{1}}^{(B, \Sigma)(A, \Xi)} \bar{s}_{\Sigma B} \bar{s}_{\Xi A}\right)
$$

Iterating the arguments, one comes to relation (148) which provides a $k$-stage gauge symmetry condition which is associated with the complete nontrivial $k$-stage NI (124). The odd graded derivation $u_{(k)}$ (149) is called the $k$-stage gauge symmetry.

Thus, components of the ascent operator $\mathbf{u}$ (139) in Theorem 33 are nontrivial gauge and higher-stage gauge symmetries. Therefore, we agree to call this operator the gauge one.

With the gauge operator (139), the extended Lagrangian $L_{e}(140)$ takes a form

$$
L_{e}=L+\mathbf{u}\left(\sum_{0 \leq k \leq N} c^{r_{k-1}} \bar{c}_{r_{k-1}}\right) \omega+L_{1}^{*}+d_{H} \sigma,
$$

where $L_{1}^{*}$ is a term of polynomial degree in antifields exceeding 1 .

The correspondence of gauge and higher-stage gauge symmetries to NI and higher-stage NI in Theorem 33 is unique due to the following direct second Noether theorem.

Theorem 34. (i) If $u$ (144) is a gauge symmetry, the variational derivative of the $d_{H^{-}}$exact density $u^{A} \mathscr{E}_{A} \omega$ (142) with respect to ghosts $c^{r}$ leads to the equality

$$
\begin{aligned}
\delta_{r}\left(u^{A} \mathscr{E}_{A} \omega\right) & =\sum(-1)^{|\Lambda|} d_{\Lambda}\left[u_{r}^{A \Lambda} \mathscr{E}_{A}\right] \\
& =\sum(-1)^{|\Lambda|} d_{\Lambda}\left(\eta\left(\Delta_{r}^{A}\right)^{\Lambda} \mathscr{E}_{A}\right) \\
& =\sum(-1)^{|\Lambda|} \eta\left(\eta\left(\Delta_{r}^{A}\right)\right)^{\Lambda} d_{\Lambda} \mathscr{E}_{A}=0,
\end{aligned}
$$

which reproduces the complete NI (99) by means of relation (137).

(ii) Given the $k$-stage gauge symmetry condition (148), the variational derivative of equality (147) with respect to ghosts $c^{r_{k}}$ leads to the equality, reproducing the $k$-stage NI (124) by means of relations (135)-(137).

Remark 35. One can consider gauge symmetries which need not be linear in ghosts. However, direct second Noether Theorem 34 is not relevant to these gauge symmetries because, in this case, an Euler-Lagrange operator satisfies the identities depending on ghosts. 


\section{Lagrangian BRST Theory}

In contrast with the KT operator (121), the gauge operator $\mathbf{u}$ (138) need not be nilpotent. Let us study its extension to a nilpotent graded derivation

$$
\begin{aligned}
\mathbf{b}= & \mathbf{u}+\gamma=\mathbf{u}+\sum_{1 \leq k \leq N+1} \gamma^{(k)}=\mathbf{u}+\sum_{1 \leq k \leq N+1} \gamma^{r_{k-1}} \frac{\partial}{\partial c^{r_{k-1}}} \\
= & \left(u^{A} \frac{\partial}{\partial s^{A}}+\gamma^{r} \frac{\partial}{\partial c^{r}}\right) \\
& +\sum_{0 \leq k \leq N-1}\left(u^{r_{k}} \frac{\partial}{\partial c^{r_{k}}}+\gamma^{r_{k+1}} \frac{\partial}{\partial c^{r_{k+1}}}\right)
\end{aligned}
$$

of ghost number 1 by means of antifield-free terms $\gamma^{(k)}$ of higher polynomial degree in ghosts $c^{r_{i}}$ and their jets $c_{\Lambda}^{r_{i}}, 0 \leq$ $i<k$. We call $\mathbf{b}$ (155) the BRST operator, where $k$-stage gauge symmetries are extended to $k$-stage BRST transformations acting both on $(k-1)$-stage and $k$-stage ghosts [18]. If a BRST operator exists, sequence (138) is brought into a BRST complex

$$
\begin{aligned}
& 0 \longrightarrow \mathcal{S}_{\infty}^{0, n}[F ; Y] \stackrel{\mathbf{b}}{\longrightarrow} P_{\infty}^{0, n}\{N\}^{1} \stackrel{\mathbf{b}}{\longrightarrow} P_{\infty}^{0, n}\{N\}^{2} \\
& \quad \stackrel{\mathbf{b}}{\longrightarrow} \cdots .
\end{aligned}
$$

There is the following necessary condition of the existence of such a BRST extension.

Theorem 36. The gauge operator (138) admits the nilpotent extension (155) only if the gauge symmetry conditions (148) and the higher-stage NI (124) are satisfied off-shell.

Proof. It is easily justified that if the graded derivation $\mathbf{b}$ (155) is nilpotent, then the right-hand sides of equalities (148) equal zero; that is,

$$
u^{(k+1)}\left(u^{(k)}\right)=0, \quad 0 \leq k \leq N-1, u^{(0)}=u .
$$

Using relations (134)-(137), one can show that, in this case, the right-hand sides of the higher-stage NI (124) also equal zero [2]. It follows that the summand $G_{r_{k}}$ of each cocycle $\Delta_{r_{k}}(122)$ is $\delta_{k-1}$-closed. Then its summand $h_{r_{k}}$ also is $\delta_{k-1}{ }^{-}$ closed and, consequently, $\delta_{k-2}$-closed. Hence it is $\delta_{k-1}$-exact by virtue of Condition 1 . Therefore, $\Delta_{r_{k}}$ contains only the term $G_{r_{k}}$ linear in antifields.

It follows at once from equalities (157) that the higherstage gauge operator

$$
u_{\mathrm{HS}}=\mathbf{u}-u=u^{(1)}+\cdots+u^{(N)}
$$

is nilpotent, and $\mathbf{u}(\mathbf{u})=u(\mathbf{u})$. Therefore, the nilpotency condition for the BRST operator $\mathbf{b}$ (155) takes a form

$$
\mathbf{b}(\mathbf{b})=(u+\gamma)(\mathbf{u})+\left(u+u_{\mathrm{HS}}+\gamma\right)(\gamma)=0 .
$$

Let us denote

$$
\begin{aligned}
& \gamma^{(0)}=0, \\
& \gamma^{(k)}=\gamma_{(2)}^{(k)}+\cdots+\gamma_{(k+1)}^{(k)}, \quad k=1, \ldots, N+1, \\
& \gamma_{(i)}^{r_{k-1}} \\
& =\sum_{k_{1}+\cdots+k_{i}=k+1-i}\left(\sum_{0 \leq\left|\Lambda_{k_{j}}\right|} \gamma_{(i) r_{k_{1}}, \ldots, r_{k_{i}}}^{r_{k_{1}}, \Lambda_{k_{1}}, \ldots, \Lambda_{k_{i}}} c_{\Lambda_{k_{1}}}^{r_{k_{1}}} \ldots c_{\Lambda_{k_{i}}}^{r_{k_{i}}}\right), \\
& \gamma^{(N+2)}=0,
\end{aligned}
$$

where $\gamma_{(i)}^{(k)}$ are terms of polynomial degree $2 \leq i \leq k+1$ in ghosts. Then the nilpotent property (159) of $\mathbf{b}$ falls into a set of equalities

$$
\begin{aligned}
& u^{(k+1)}\left(u^{(k)}\right)=0, \quad 0 \leq k \leq N-1, \\
& \left(u+\gamma_{(2)}^{(k+1)}\right)\left(u^{(k)}\right)+u_{\mathrm{HS}}\left(\gamma_{(2)}^{(k)}\right)=0, \quad 0 \leq k \leq N+1, \\
& \gamma_{(i)}^{(k+1)}\left(u^{(k)}\right)+u\left(\gamma_{(i-1)}^{(k)}\right)+u_{\mathrm{HS}}\left(\gamma_{(i)}^{k}\right) \\
& \quad+\sum_{2 \leq m \leq i-1} \gamma_{(m)}\left(\gamma_{(i-m+1)}^{(k)}\right)=0, \quad i-2 \leq k \leq N+1,
\end{aligned}
$$

of ghost polynomial degrees 1,2 , and $3 \leq i \leq N+3$, respectively.

Equalities (161) are exactly the gauge symmetry conditions (157) in Theorem 36.

Equality (162) for $k=0$ reads

$$
\begin{aligned}
\left(u+\gamma^{(1)}\right)(u) & =0 \\
\sum\left(d_{\Lambda}\left(u^{A}\right) \partial_{A}^{\Lambda} u^{B}+d_{\Lambda}\left(\gamma^{r}\right) u_{r}^{B, \Lambda}\right) & =0 .
\end{aligned}
$$

It takes a form of the Lie antibracket

$$
[u, u]=-2 \gamma^{(1)}(u)=-2 \sum d_{\Lambda}\left(\gamma^{r}\right) u_{r}^{B, \Lambda} \partial_{B}
$$

of an odd gauge symmetry $u$. Its right-hand side factorizes through $u$, but it is nonlinear in ghosts.

Equalities (162)-(163) for $k=1$ take a form

$$
\begin{aligned}
& \left(u+\gamma_{(2)}^{(2)}\right)\left(u^{(1)}\right)+u^{(1)}\left(\gamma^{(1)}\right)=0, \\
& \gamma_{(3)}^{(2)}\left(u^{(1)}\right)+\left(u+\gamma^{(1)}\right)\left(\gamma^{(1)}\right)=0 .
\end{aligned}
$$

In particular, if a Lagrangian system is irreducible, that is, $u^{(k)}=0$, the BRST operator reads

$$
\begin{aligned}
\mathbf{b} & =u+\gamma^{(1)}=u^{A} \partial_{A}+\gamma^{r} \partial_{r} \\
& =\sum u_{r}^{A, \Lambda} c_{\Lambda}^{r} \partial_{A}+\sum \gamma_{p q}^{r, \Lambda, \Xi} c_{\Lambda}^{p} c_{\Xi}^{q} \partial_{r} .
\end{aligned}
$$

In this case, the nilpotency conditions (166) are reduced to the equality

$$
\left(u+\gamma^{(1)}\right)\left(\gamma^{(1)}\right)=0 .
$$


Furthermore, let a gauge symmetry $u$ be affine in fields $s^{A}$ and their jets. It follows from the nilpotency condition (164) that the BRST term $\gamma^{(1)}$ is independent of original fields and their jets. Then relation (168) takes a form of the Jacobi identity

$$
\gamma^{(1)}\left(\gamma^{(1)}\right)=0
$$

for coefficient functions $\gamma_{p q}^{r, \Lambda, \Xi}(x)$ in the Lie antibracket (165).

Relations (165) and (169) motivate us to think of equalities (162)-(163) in a general case of reducible gauge symmetries as being sui generis generalized commutation relations and Jacobi identities of gauge symmetries, respectively [18]. Therefore, one can say that gauge symmetries are algebraically closed (in the terminology of [19]) if the gauge operator $\mathbf{u}$ (139) admits the nilpotent BRST extension $\mathbf{b}$ (155).

The DBGA $\mathscr{P}_{\infty}^{*}\{N\}$ (133) is a particular field-antifield theory of the following type $[2,15,19]$.

Let us consider a pull-back composite bundle

$$
W=\underset{X}{Z} \underset{X}{\times} Z^{\prime} \longrightarrow Z \longrightarrow X
$$

where $Z^{\prime} \rightarrow X$ is a vector bundle. Let us regard it as an odd graded vector bundle over $Z$. The density-dual $\overline{V W}$ of the vertical tangent bundle $V W$ of $W \rightarrow X$ is a graded vector bundle

$$
\overline{V W}=\left(\left(\overline{Z^{\prime}} \bigoplus_{Z} V^{*} Z\right) \bigoplus_{Z} \bigwedge^{n} T^{*} X\right) \bigoplus_{Y} Z^{\prime}
$$

over $Z$ (cf. (90)). Let us consider the DBGA $\mathscr{P}_{\infty}^{*}[\overline{V W} ; Z](92)$ with the local generating basis $\left(z^{a}, \bar{z}_{a}\right),\left[\bar{z}_{a}\right]=\left[z^{a}\right]+1$. Its elements $z^{a}$ and $\bar{z}_{a}$ are called fields and antifields, respectively.

Graded densities of this DBGA are endowed with the antibracket

$$
\left\{\mathfrak{Q} \omega, \mathfrak{\Omega}^{\prime} \omega\right\}=\left[\frac{\overleftarrow{\delta} \mathfrak{Q}}{\delta \bar{z}_{a}} \frac{\delta \mathfrak{Q}^{\prime}}{\delta z^{a}}+(-1)^{\left[\mathfrak{Q}^{\prime}\right]\left(\left[\mathfrak{Q}^{\prime}\right]+1\right)} \frac{\overleftarrow{\delta} \mathfrak{Q}^{\prime}}{\delta \bar{z}_{a}} \frac{\delta \mathfrak{Q}}{\delta z^{a}}\right] \omega .
$$

Then one associates with any (even) Lagrangian $\mathfrak{Q} \omega$ the odd vertical graded derivations

$$
\begin{aligned}
& v_{\mathfrak{Q}}=\overleftarrow{\mathscr{E}}^{a} \partial_{a}=\frac{\overleftarrow{\delta} \mathfrak{Q}}{\delta \bar{z}_{a}} \frac{\partial}{\partial z^{a}}, \\
& \bar{v}_{\mathfrak{L}}=\overleftarrow{\partial}^{a} \mathscr{E}_{a}=\frac{\overleftarrow{\partial}}{\partial \bar{z}_{a}} \frac{\delta \mathfrak{L}}{\delta z^{a}}, \\
& \vartheta_{\mathfrak{L}}=v_{\mathfrak{Q}}+\bar{v}_{\mathfrak{L}}^{l}=(-1)^{[a]+1}\left(\frac{\delta \mathfrak{Q}}{\delta \bar{z}^{a}} \frac{\partial}{\partial z_{a}}+\frac{\delta \mathfrak{Q}}{\delta z^{a}} \frac{\partial}{\partial \bar{z}_{a}}\right),
\end{aligned}
$$

such that $\vartheta_{\mathfrak{Q}}\left(\mathbf{\Omega}^{\prime} \omega\right)=\left\{\mathfrak{\Omega} \omega, \mathbf{\Omega}^{\prime} \omega\right\}$.

Theorem 37. The following conditions are equivalent $[2,12]$.

(i) The antibracket of a Lagrangian $\mathfrak{\Omega} \omega$ is $d_{H^{-}}$exact; that is,

$$
\{\mathfrak{\Omega} \omega, \mathfrak{\Omega} \omega\}=2 \frac{\overleftarrow{\delta} \mathfrak{Q}}{\delta \bar{z}_{a}} \frac{\delta \mathfrak{Q}}{\delta z^{a}} \omega=d_{H} \sigma
$$

(ii) The graded derivation $\vartheta_{\mathfrak{Q}}(174)$ is nilpotent.

Equality (175) is called the classical master equation. A solution of the master equation (175) is called nontrivial if both derivations (173) do not vanish.

Being an element of the DBGA $\mathscr{P}_{\infty}^{*}\{N\}$ (133), an original Lagrangian $L$ obeys the master equation (175) and yields the graded derivations $v_{L}=0, \bar{v}_{L}=\bar{\delta}$ (173); that is, it is a trivial solution of the master equation. However, its extension $L_{e}$ (153) need not satisfy the master equation. Therefore, let us consider its extension

$$
L_{E}=L_{e}+L^{\prime}=L+L_{1}+L_{2}+\cdots
$$

by means of even densities $L_{i}, i \geq 2$, of zero antifield number and polynomial degree $i$ in ghosts. Then the following is a corollary of Theorem 37.

Corollary 38. A Lagrangian $L$ is extended to a proper solution $L_{E}$ (176) of the master equation iff the gauge operator $\mathbf{u}$ (138) admits a nilpotent extension $\vartheta_{E}(174)$.

However, one can say something more $[2,12]$.

Theorem 39. If the gauge operator $\mathbf{u}$ (138) can be extended to the BRST operator $\mathbf{b}$ (155), then the master equation has a nontrivial proper solution

$$
\begin{aligned}
L_{E} & =L_{e}+\sum_{1 \leq k \leq N} \gamma^{r_{k-1}} \bar{c}_{r_{k-1}} \omega \\
& =L+\mathbf{b}\left(\sum_{0 \leq k \leq N} c^{r_{k-1}} \bar{c}_{r_{k-1}}\right) \omega+d_{H} \sigma,
\end{aligned}
$$

such that $\mathbf{b}=v_{E}$ is the graded derivation defined by the Lagrangian $L_{E}$ (177).

The Lagrangian $L_{E}(177)$ is said to be the BRST extension of an original Lagrangian $L$.

\section{Example: Topological BF Theory}

We address the topological BF theory of two exterior forms $A$ and $B$ of form degree $|A|+|B|=\operatorname{dim} X-1$ on a smooth manifold $X[20,27]$. It is reducible degenerate Lagrangian theory which satisfies homology regularity condition (Condition 1). Its dynamic variables $A$ and $B$ are sections of a fibre bundle

$$
\begin{aligned}
Y & =\bigwedge^{p} T^{*} X \oplus \bigwedge^{q} T^{*} X, \\
p+q & =n-1>1,
\end{aligned}
$$

coordinated by $\left(x^{\lambda}, A_{\mu_{1} \cdots \mu_{p}}, B_{v_{1} \cdots v_{q}}\right)$. Without loss of generality, let $q$ be even and $q \geq p$. The corresponding differential graded algebra is $\mathcal{O}_{\infty}^{*}(41)$.

There are canonical $p$ - and $q$-forms

$$
\begin{aligned}
& A=A_{\mu_{1} \cdots \mu_{p}} d x^{\mu_{1}} \wedge \cdots \wedge d x^{\mu_{p}}, \\
& B=B_{\nu_{1} \cdots \nu_{q}} d x^{\nu_{1}} \wedge \cdots \wedge d x^{\nu_{q}}
\end{aligned}
$$


on $Y$. A Lagrangian of topological BF theory reads

$$
L_{\mathrm{BF}}=A \wedge d_{H} B=\epsilon^{\mu_{1} \cdots \mu_{n}} A_{\mu_{1} \cdots \mu_{p}} d_{\mu_{p+1}} B_{\mu_{p+2} \cdots \mu_{n}} \omega \text {, }
$$

where $\epsilon$ is the Levi-Civita symbol. It is a reduced first order Lagrangian. Its first order Euler-Lagrange operator

$$
\begin{aligned}
\delta L= & \mathscr{E}_{A}^{\mu_{1} \cdots \mu_{p}} d A_{\mu_{1} \cdots \mu_{p}} \wedge \omega+\mathscr{E}_{B}^{v_{p+2} \cdots v_{n}} d B_{v_{p+2} \cdots v_{n}} \\
& \wedge \omega \\
\mathscr{E}_{A}^{\mu_{1} \cdots \mu_{p}}= & \epsilon^{\mu_{1} \cdots \mu_{n}} d_{\mu_{p+1}} B_{\mu_{p+2} \cdots \mu_{n}}, \\
\mathscr{E}_{B}^{\mu_{p+2} \cdots \mu_{n}}= & -\epsilon^{\mu_{1} \cdots \mu_{n}} d_{\mu_{p+1}} A_{\mu_{1} \cdots \mu_{p}}
\end{aligned}
$$

satisfies the Noether identities

$$
\begin{aligned}
& d_{\mu_{1}} \mathscr{E}_{A}^{\mu_{1} \cdots \mu_{p}}=0, \\
& d_{v_{1}} \mathscr{E}_{B}^{\nu_{1} \cdots v_{q}}=0 .
\end{aligned}
$$

Given a family of vector bundles

$$
\begin{aligned}
E_{k} & =\bigwedge^{p-k-1} T^{*} X \underset{X}{q^{-k-1}} \bigwedge^{*} T^{*} X, \quad 0 \leq k<p-1, \\
E_{k} & =\underset{R}{\mathbb{R}} \bigwedge^{q-p} T^{*} X, \quad k=p-1, \\
E_{k} & =\bigwedge^{q-k-1} T^{*} X, \quad p-1<k<q-1, \\
E_{q-1} & =X \times \mathbb{R},
\end{aligned}
$$

let us enlarge an original differential graded algebra $\mathcal{O}_{\infty}^{*}$ to the BGDA $\mathscr{P}_{\infty}^{*}\{q-1\}$ (133) which is

$$
\begin{aligned}
& \mathscr{P}_{\infty}^{*}\{q-1\}=\mathscr{P}_{\infty}^{*}\left[\overline{V Y} \bigoplus_{Y} E_{0}\right. \\
& \left.\oplus \cdots \bigoplus_{Y} E_{q-1} \bigoplus_{Y} \bar{E}_{0} \bigoplus_{Y} \cdots \bigoplus_{Y} \bar{E}_{q-1} ; Y\right] .
\end{aligned}
$$

It possesses a local generating basis

$$
\begin{aligned}
& \left\{A_{\mu_{1} \cdots \mu_{p}}, B_{\nu_{1} \cdots v_{q}}, \varepsilon_{\mu_{2} \cdots \mu_{p}}, \ldots, \varepsilon_{\mu_{p}}, \varepsilon, \xi_{\nu_{2} \cdots v_{q}}, \ldots, \xi_{v_{q}}, \xi\right. \\
& \left.\bar{A}^{\mu_{1} \cdots \mu_{p}}, \bar{B}^{\nu_{1} \cdots v_{q}}, \bar{\varepsilon}^{\mu_{2} \cdots \mu_{p}}, \ldots, \bar{\varepsilon}^{\mu_{p}}, \bar{\varepsilon}, \bar{\xi}^{\nu_{2} \cdots v_{q}}, \ldots, \bar{\xi}^{v_{q}}, \bar{\xi}\right\}
\end{aligned}
$$

of Grassmann parity

$$
\begin{aligned}
& {\left[\varepsilon_{\mu_{k} \cdots \mu_{p}}\right]=\left[\xi_{v_{k} \cdots v_{q}}\right]=(k+1) \bmod 2,} \\
& {[\varepsilon]=p \bmod 2,[\xi]=0,} \\
& {\left[\bar{\varepsilon}^{\mu_{k} \cdots \mu_{p}}\right]=\left[\bar{\xi}^{\nu_{k} \cdots \nu_{q}}\right]=k \bmod 2,}
\end{aligned}
$$

$$
[\bar{\varepsilon}]=(p+1) \bmod 2,[\bar{\xi}]=1
$$

of ghost number

$$
\begin{aligned}
& \operatorname{gh}\left[\varepsilon_{\mu_{k} \cdots \mu_{p}}\right]=\operatorname{gh}\left[\xi_{v_{k} \cdots v_{q}}\right]=k, \\
& \operatorname{gh}[\varepsilon]=p+1, \operatorname{gh}[\xi]=q+1,
\end{aligned}
$$

and of antifield number

$$
\begin{aligned}
\text { Ant }\left[\bar{A}^{\mu_{1} \cdots \mu_{p}}\right] & =\text { Ant }\left[\bar{B}^{v_{p+1} \cdots v_{q}}\right]=1, \\
\text { Ant }\left[\bar{\varepsilon}^{\mu_{k} \cdots \mu_{p}}\right] & =\text { Ant }\left[\bar{\xi}^{v_{k} \cdots v_{q}}\right]=k+1, \\
\text { Ant }[\bar{\varepsilon}] & =p, \\
\text { Ant }[\bar{\varepsilon}] & =q .
\end{aligned}
$$

One can show that homology regularity condition (Condition 1 ) holds ([20, Lemma 4.5.5]), and the DBGA $\mathscr{P}_{\infty}^{*}\{q-1\}$ (184) is endowed with the Koszul-Tate operator

$$
\begin{aligned}
& \delta_{\mathrm{KT}}=\frac{\overleftarrow{\partial}}{\partial \bar{A}^{\mu_{1} \cdots \mu_{p}}} \mathscr{E}_{A}^{\mu_{1} \cdots \mu_{p}}+\frac{\overleftarrow{\partial}}{\partial \bar{B}^{\nu_{1} \cdots v_{q}}} \mathscr{E}_{B}^{\nu_{1} \cdots v_{q}} \\
& +\sum_{2 \leq k \leq p} \frac{\overleftarrow{\partial}}{\partial \bar{\varepsilon}^{\mu_{k} \cdots \mu_{p}}} \Delta_{A}^{\mu_{k} \cdots \mu_{p}}+\frac{\overleftarrow{\partial}}{\partial \bar{\varepsilon}} d_{\mu_{p}} \bar{\varepsilon}^{\mu_{p}} \\
& +\sum_{2 \leq k \leq q} \frac{\overleftarrow{\partial}}{\partial \bar{\xi}^{\nu_{k} \cdots v_{q}}} \Delta_{B}^{v_{k} \cdots v_{q}}+\frac{\overleftarrow{\partial}}{\partial \bar{\xi}} d_{v_{q}} \bar{\xi}^{v_{q}}, \\
& \Delta_{A}^{\mu_{2} \cdots \mu_{p}}=d_{\mu_{1}} \bar{A}^{\mu_{1} \cdots \mu_{p}}, \\
& \Delta_{A}^{\mu_{k+1} \cdots \mu_{p}}=d_{\mu_{k}} \bar{\varepsilon}^{\mu_{k} \mu_{k+1} \cdots \mu_{p}}, \\
& 2 \leq k<p \\
& \Delta_{B}^{\nu_{2} \cdots v_{q}}=d_{\nu_{1}} \bar{B}^{\nu_{1} \cdots v_{q}}, \\
& \Delta_{B}^{v_{k+1} \cdots v_{q}}=d_{v_{k}} \bar{\xi}^{v_{k} v_{k+1} \cdots v_{q}} \text {, } \\
& 2 \leq k<q .
\end{aligned}
$$

Its nilpotentness provides the complete Noether identities $(182)$ and the $(k-1)$-stage ones

$$
\begin{aligned}
& d_{\mu_{k}} \Delta_{A}^{\mu_{k} \cdots \mu_{p}}=0, \quad k=2, \ldots, p, \\
& d_{\nu_{k}} \Delta_{B}^{\nu_{k} \cdots v_{q}}=0, \quad k=2, \ldots, q .
\end{aligned}
$$

It follows that the topological BF theory is $(q-1)$-reducible.

Applying inverse second Noether Theorem 33, one obtains the gauge operator (139) which reads

$$
\begin{aligned}
\mathbf{u}= & d_{\mu_{1}} \varepsilon_{\mu_{2} \cdots \mu_{p}} \frac{\partial}{\partial A_{\mu_{1} \mu_{2} \cdots \mu_{p}}}+d_{\nu_{1}} \xi_{\nu_{2} \cdots v_{q}} \frac{\partial}{\partial B_{v_{1} \nu_{2} \cdots v_{q}}} \\
& +\left[d_{\mu_{2}} \varepsilon_{\mu_{3} \cdots \mu_{p}} \frac{\partial}{\partial \varepsilon_{\mu_{2} \mu_{3} \cdots \mu_{p}}}+\cdots+d_{\mu_{p}} \varepsilon \frac{\partial}{\partial \varepsilon_{\mu_{p}}}\right] \\
& +\left[d_{v_{2}} \xi_{v_{3} \cdots v_{q}} \frac{\partial}{\partial \xi_{v_{2} \nu_{3} \cdots v_{q}}}+\cdots+d_{v_{q}} \xi \frac{\partial}{\partial \xi_{v_{q}}}\right] .
\end{aligned}
$$


In particular, a gauge symmetry of the Lagrangian $L_{\mathrm{BF}}(180)$ is

$$
u=d_{\mu_{1}} \varepsilon_{\mu_{2} \cdots \mu_{p}} \frac{\partial}{\partial A_{\mu_{1} \mu_{2} \cdots \mu_{p}}}+d_{\nu_{1}} \xi_{\nu_{2} \cdots v_{q}} \frac{\partial}{\partial B_{v_{1} \nu_{2} \cdots v_{q}}} .
$$

It also is readily observed that the gauge operator $\mathbf{u}$ (191) is nilpotent. Thus, it is the BRST operator $\mathbf{b}=\mathbf{u}$. As a result, the Lagrangian $L_{\mathrm{BF}}$ is extended to the proper solution of the master equation $L_{E}=L_{e}(177)$ which reads

$$
\begin{aligned}
L_{e}= & L_{\mathrm{BF}}+\varepsilon_{\mu_{2} \cdots \mu_{p}} d_{\mu_{1}} \bar{A}^{\mu_{1} \cdots \mu_{p}}+\sum_{1<k<p} \varepsilon_{\mu_{k+1} \cdots \mu_{p}} d_{\mu_{k}} \bar{\varepsilon}^{\mu_{k} \cdots \mu_{p}} \\
& +\varepsilon d_{\mu_{p}} \bar{\varepsilon}^{\mu_{p}}+\xi_{v_{2} \cdots v_{q}} d_{v_{1}} \bar{B}^{\nu_{1} \cdots v_{q}} \\
& +\sum_{1<k<q} \xi_{v_{k+1} \cdots v_{q}} d_{v_{k}} \bar{\xi}^{v_{k} \cdots \mu_{q}}+\xi d_{v_{q}} \bar{\xi}^{v_{q}} .
\end{aligned}
$$

\section{Conflict of Interests}

The author declares that there is no conflict of interests regarding the publication of this paper.

\section{References}

[1] Y. Kosmann-Schwarzbach, The Noether Theorems. Invariance and the Conservation Laws in the Twentieth Century, Springer, 2011.

[2] D. Bashkirov, G. Giachetta, L. Mangiarotti, and G. Sardanashvily, "The KT-BRST complex of a degenerate Lagrangian system," Letters in Mathematical Physics, vol. 83, no. 3, pp. 237$252,2008$.

[3] G. Sardanashvily, Advanced Differential Geometry for Theoreticians. Fiber Bundles, Jet Manifolds and Lagrangian Theory, Lambert Academic Publishing, Saarbrücken, Germany, 2013.

[4] P. J. Olver, Applications of Lie Groups to Differential Equations, Springer, New York, NY, USA, 1986.

[5] F. Takens, "A global version of the inverse problem of the calculus of variations," Journal of Differential Geometry, vol. 14, no. 4, pp. 543-562, 1979.

[6] R. L. Bryant, S. S. Chern, R. B. Gardner, H. L. Goldschmidt, and P. A. Griffiths, Exterior Differential Systems, Springer, 1991.

[7] I. Krasil'shchik, V. Lychagin, and A. Vinogradov, Geometry of Jet Spaces and Nonlinear Partial Differential Equations, Gordon and Breach, Glasgow, Scotland, 1985.

[8] J. F. Cariñena and H. Figueroa, "Singular Lagrangians in supermechanics," Differential Geometry and Its Applications, vol. 18, no. 1, pp. 33-46, 2003.

[9] R. Cianci, M. Francaviglia, and I. Volovich, "Variational calculus and Poincaré-Cartan formalism on supermanifolds," Journal of Physics A, vol. 28, no. 3, pp. 723-734, 1995.

[10] D. H. Franco and C. M. Polito, "Supersymmetric field-theoretic models on a supermanifold," Journal of Mathematical Physics, vol. 45, no. 4, pp. 1447-1473, 2004.

[11] J. Monterde, J. M. Masqué, and J. A. Vallejo, "The PoincaréCartan form in superfield theory," International Journal of Geometric Methods in Modern Physics, vol. 3, no. 4, pp. 775-822, 2006.
[12] G. Sardanashvily, “Graded Lagrangian formalism," International Journal of Geometric Methods in Modern Physics, vol. 10, no. 5, Article ID 1350016, 37 pages, 2013.

[13] C. Bartocci, U. Bruzzo, and D. Hernández Ruipérez, The Geometry of Supermanifolds, Kluwer, Dordrecht, The Netherlands, 1991.

[14] I. Anderson, "Introduction to the variational bicomplex," Contemporary Mathematics, vol. 132, p. 51, 1992.

[15] G. Barnich, F. Brandt, and M. Henneaux, "Local BRST cohomology in gauge theories," Physics Reports, vol. 338, no. 5, pp. 439-569, 2000.

[16] G. Giachetta, L. Mangiarotti, and G. Sardanashvily, "Lagrangian supersymmetries depending on derivatives. Global analysis and cohomology," Communications in Mathematical Physics, vol. 259, no. 1, pp. 103-128, 2005.

[17] R. Fulp, T. Lada, and J. Stasheff, "Sh-Lie algebras induced by gauge transformations," Communications in Mathematical Physics, vol. 231, no. 1, pp. 25-43, 2002.

[18] G. Giachetta, L. Mangiarotti, and G. Sardanashvily, "On the notion of gauge symmetries of generic Lagrangian field theory," Journal of Mathematical Physics, vol. 50, no. 1, Article ID 012903, 19 pages, 2009.

[19] J. Gomis, J. París, and S. Samuel, "Antibracket, antifields and gauge-theory quantization," Physics Reports, vol. 259, no. 1-2, pp. $1-145,1995$.

[20] G. Giachetta, L. Mangiarotti, and G. Sardanashvily, Advanced Classical Field Theory, World Scientific Publishing, Hackensack, NJ, USA, 2009.

[21] D. B. Fuks, Cohomology of Infinite-Dimensional Lie Algebras, Consultants Bureau, New York, NY, USA, 1986.

[22] M. Batchelor, “The structure of supermanifolds," Transactions of the American Mathematical Society, vol. 253, pp. 329-338, 1979.

[23] G. Sardanashvily, "Graded infinite order jet manifolds," International Journal of Geometric Methods in Modern Physics, vol. 4, no. 8, pp. 1335-1362, 2007.

[24] D. Hernández Ruipérez and J. Muñoz Masqué, "Global variational calculus on graded manifolds. I. Graded jet bundles, structure 1-form and graded infinitesimal contact transformations," Journal de Mathématiques Pures et Appliquées, vol. 63, no. 3, pp. 283-309, 1984.

[25] T. Stavracou, "Theory of connections on graded principal bundles," Reviews in Mathematical Physics, vol. 10, no. 1, pp. 4779, 1998.

[26] R. Fulp, T. Lada, and J. Stasheff, "Noether variational theorem II and the BV formalism," Rendiconti del Circolo Matematico di Palermo, vol. 2, supplement 71, p. 115, 2003.

[27] D. Birmingham, M. Blau, M. Rakowski, and G. Thompson, “Topological field theory," Physics Reports, vol. 209, no. 4-5, pp. 129-340, 1991. 


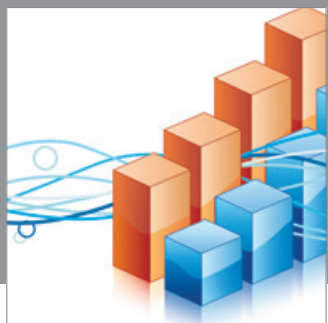

Advances in

Operations Research

mansans

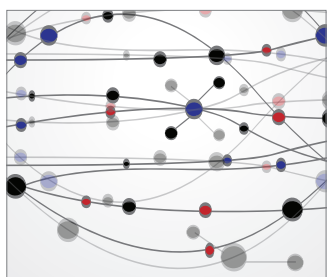

The Scientific World Journal
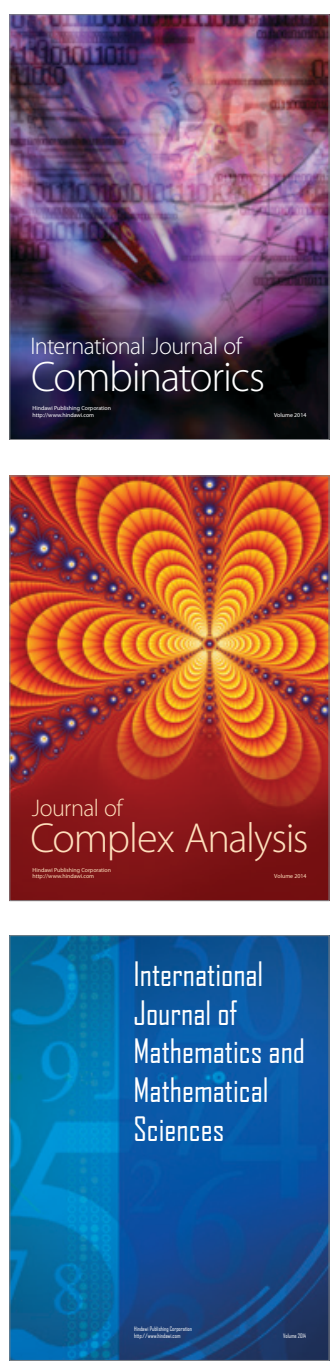
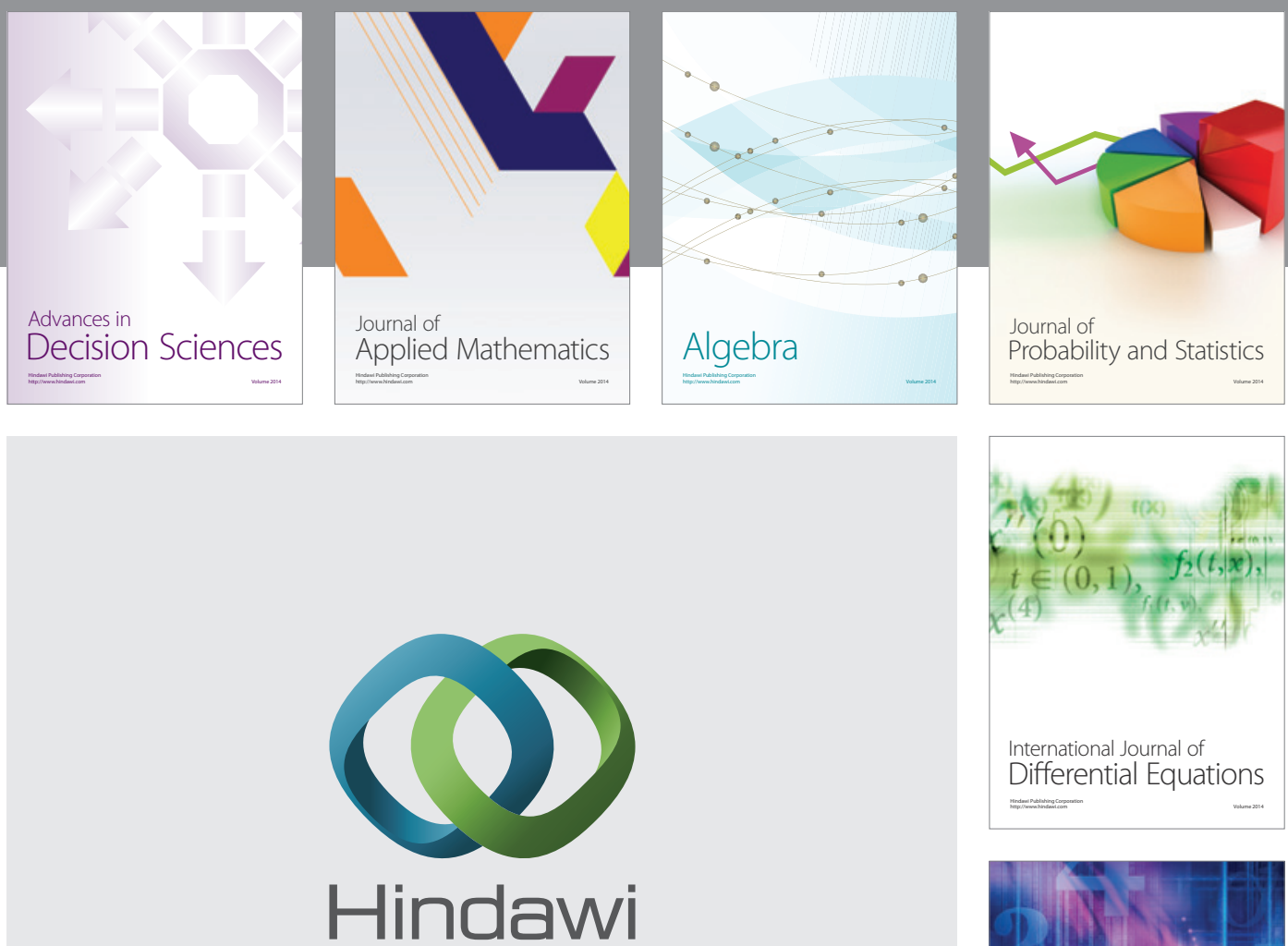

Submit your manuscripts at http://www.hindawi.com
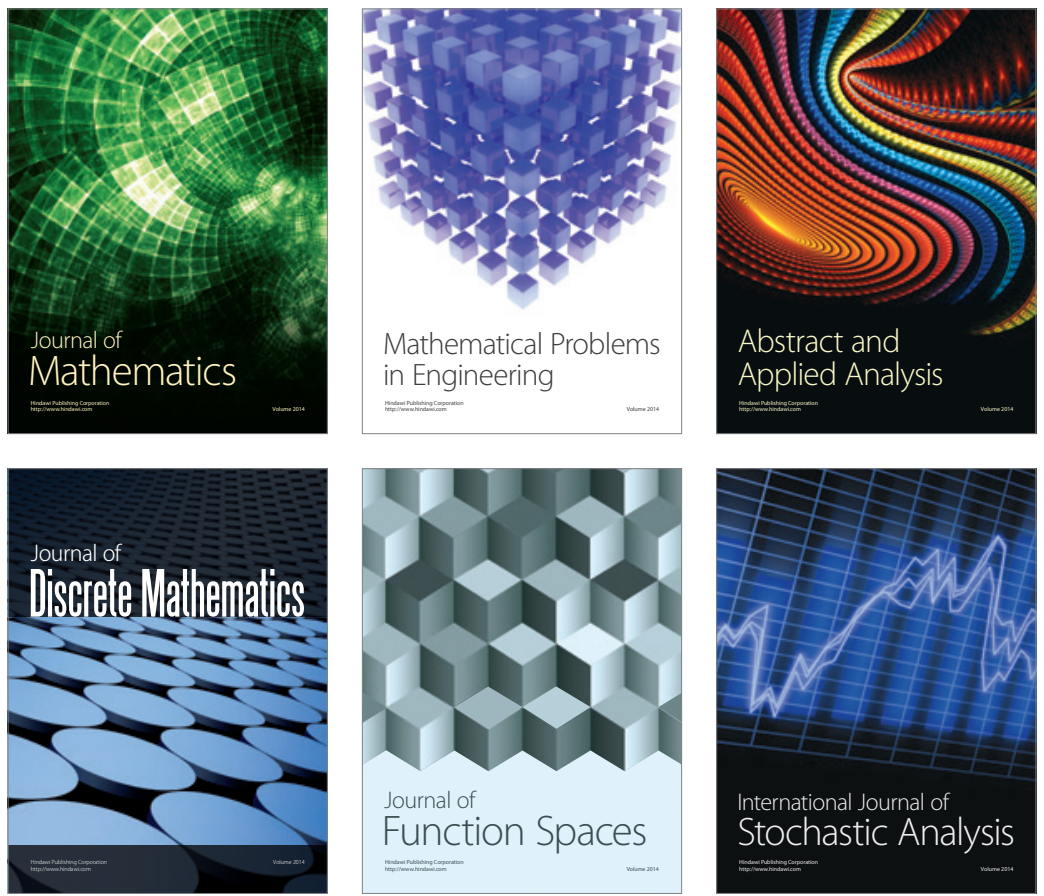

Journal of

Function Spaces

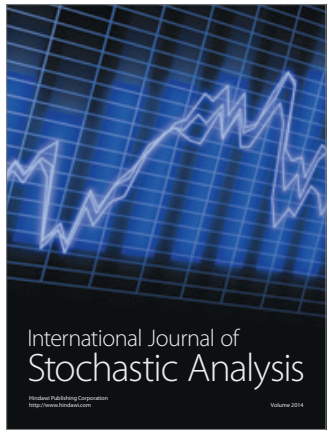

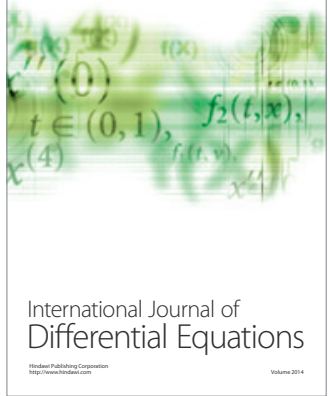
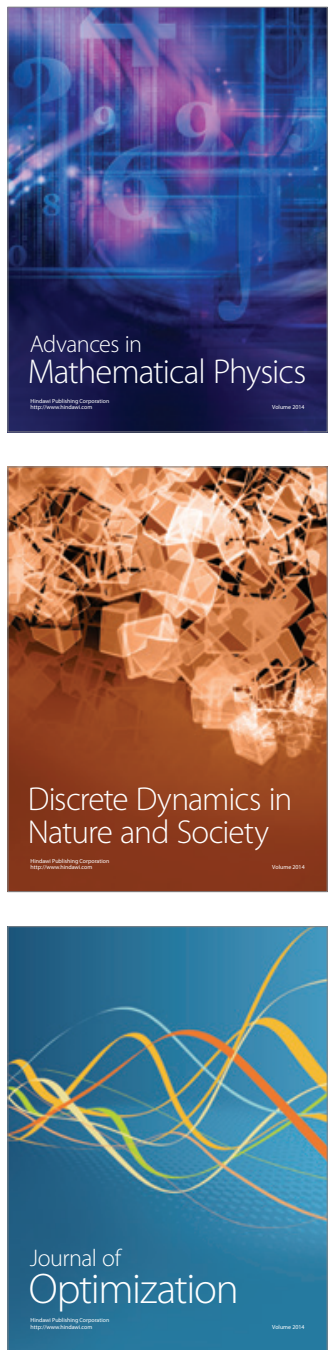